\title{
HIERARCHICAL BEAM ALIGNMENT IN SU-MIMO TERAHERTZ COMMUNICATIONS
}

\author{
Yifei $\mathrm{Wu}^{1}$, Johannes $\mathrm{Koch}^{2}$, Martin Vossiek ${ }^{2}$, and Wolfgang Gerstacker ${ }^{1}$ \\ ${ }^{1}$ Institute for Digital Communications, Friedrich-Alexander-Universität Erlangen-Nürnberg, Erlangen, Germany, \\ ${ }^{2}$ Institute of Microwaves and Photonics, Friedrich-Alexander-Universität Erlangen-Nürnberg, Erlangen, Germany
}

NOTE: Corresponding author: Yifei Wu, yifei.wu@fau.de

\begin{abstract}
Single-Carrier Frequency Division Multiple Access (SC-FDMA) is a promising technique for high data rate indoor Terahertz (THz) communications in future beyond $5 \mathrm{G}$ systems. In an indoor propagation scenario, the Line-Of-Sight (LOS) component may be blocked by the obstacles. Thus, efficient THz SC-FDMA communications require a fast and reliable Beam Alignment (BA) method for both LOS and Non-Line-Of-Sight (NLOS) scenarios. In this paper, we first adopt the hierarchical discrete Fourier transform codebook for LOS BA, and introduce the hierarchical $k$-means codebookfor NLOS BA to improve the beamforming gain. Simulation results illustrate that the hierarchical DFT codebook and the hierarchical $k$-means codebook can achieve the beamforming gain close to that of the maximum ratio transmission in LOS and NLOS cases, respectively. Based on these two codebooks, we propose a Multi-Armed Bandit (MAB) algorithm named Hierarchical Beam Alignment (HBA) for single-user SC-FDMA THz systems to reduce the BA latency. HBA utilizes a hierarchical structure in the adopted codebook and prior knowledge regarding the noise power to speed up the BA process. Both theoretical analysis and simulation results indicate that the proposed BA method converges to the optimal beam with high probability for both the hierarchical DFT codebook and the hierarchical k-means codebook in the LOS and NLOS scenarios, respectively. The latency introduced by HBA is significantly lower when compared to an exhaustive search method and other MAB-based methods.
\end{abstract}

Keywords - Beam alignment, codebook design, SC-FDMA, SU-MIMO, terahertz communications

\section{INTRODUCTION}

Terahertz (THz) communications are a key technology for the future wireless communications (beyond 5G) owing to its ample frequency spectrum resource between 0.1 and $10 \mathrm{THz}$ promising a much higher capacity than mmWave communications [1], [2]. Major breakthroughs in hardware and theory have been achieved for the efficient realization of $\mathrm{THz}$ range transmission [3], [1]. Besides the ultra-high bandwidth, $\mathrm{THz}$ wireless technology has the advantage that it could be deployed much faster and more efficiently than optical fiber systems, especially in a high-density urban environment [4]. Moreover, $\mathrm{THz}$ communications are highly suited to the indoor environ- ment since $\mathrm{THz}$ communications systems can utilize the Non-LineOf-Sight (NLOS) Multipath Components (MPCs) to enhance the link quality in indoor application [5], [6]. However, NLOS MPCs result in a highly frequencyselective channel, which requires the transmission system to deal with the Inter-Symbol Interference (ISI) effect. The Single-Carrier Frequency Division Multiple Access (SC-FDMA) transmission approach provides a solution to conquer the high frequency selectivity of the channel. Compared to Orthogonal Frequency Division Multiplexing (OFDM), SC-FDMA utilizes a Discrete Fourier Transform (DFT) pre-coding to reduce Peak-toAverage Power Ratio (PAPR) [7]. Due to the high carrier

\footnotetext{
${ }^{0}$ This work has been supported by the German Research Foundation (DFG) under grant GE 861/9-1.
}

frequency in Time-Division Duplex (TDD) massive Multiple-InputMultiple-Output (MIMO) THz communications, the power amplifier efficiency in the $\mathrm{THz}$ band is degraded significantly. Hence, the benefits of a low PAPR of SC-FDMA are emphasized for $\mathrm{THz}$ communications. Moreover, $\mathrm{THz}$ signals suffer from severe path loss caused by high carrier frequency around hundreds of $\mathrm{GHz}$, which limits $\mathrm{THz}$ communications in an indoor transmission scenario [8], [6]. To overcome the high path loss, directional beamforming with massive Transmitter (Tx) and Receiver (Rx) antenna arrays is regarded as a reasonable solution. Because of the short wavelength in $\mathrm{THz}$ bands, and the progress of antenna technology, the massive antenna arrays in principle can be packed into a small area, which enables a large beamforming gain at both $\mathrm{Tx}$ and $\mathrm{Rx}$. Besides the massive antenna array, the $\mathrm{Tx}$ and $\mathrm{Rx}$ beams at the base station and user terminal must be formed accurately to achieve the beamforming gain. However, the design of beamforming codes is usually based on perfect Channel State Information (CSI), which is difficult to acquire in the $\mathrm{THz}$ case especially at the Tx side due to the large-scale antenna array and the small Signal-to-Noise Ratio (SNR) before beamforming.

One way to circumvent the CSI requirement is to employ a Beam Alignment (BA) scheme. BA is a process to find the optimal transmit-receive beam pair from predefined codebooks to maximize the receive signal strength. The beam alignment problem has been widely studied in mmWave communications. The authors in [9] advocate decoupling the BA process in mmWave transmission into two steps to reduce the BA latency caused by 
a large codebook. First, the receiver beam is set to a quasi-omnidirectional beam and the transmitter scans the beam codebook exhaustively for the best transmit beam. Then, the receiver searches for the best beam with quasi-omnidirectional transmit beam. To further accelerate the BA process, we replace the BA step at the receiver by the frequency-domain Minimum Mean-Squared Error (MMSE) equalizer design according to [10] exploiting the high SNR at the receiver after the transmit BA for channel estimation in [11]. Still, the exhaustive search at Tx may take up to several seconds with a massive transmit antenna array.

To reduce the duration of the BA process at the $\mathrm{Tx}$, Multi-Armed Bandit (MAB) theory has been advocated in many search strategies for the mmWave BA problem [12]. MAB focuses on choosing the best arm with maximal expected gain among multiple actions when each choice's gain is known after each selection. Based on the MAB-BA problem framework, many variants of MAB solutions have been adapted to the mmWave communication system. In [13], the authors proposed a celebrated upper confidence bound beam selection scheme in traditional MIMO systems. In [14], the authors introduced a beam alignment algorithm employing contextual information based on a structured MAB framework. The algorithm in [15] utilizes latent probability structure information from the varying transmission environments and promises better performance in fast varying mmWave channels. Another work proposed a distributed BA search method based on adversarial bandit theory, where Tx and Rx choose their beamforming and combining vector independently of each other [16]. Nevertheless, these works do not provide a method with a short training time and accurate beam alignment, especially in multipath channels. Hence, the authors in [9] proposed a Hierarchical Beam Alignment (HBA) algorithm based on a stochastic MAB scheme, leveraging the inherent correlation structure among beams. HBA can identify the optimal beam accurately with a short exploration time, which fulfills the fast BA requirement in narrowband MIMO LOS mmWave communications. However, no specific algorithm is proposed yet for broadband MIMO THz communications. Moreover, if the LOS component of propagation is blocked, the communications quality cannot be guaranteed by the aforementioned algorithms.

In this paper, we consider the design of HBA algorithm based on [9]. Given a hierarchical codebook, HBA selects the beams to maximize the cumulative receive power within a certain period in a hierarchical manner utilizing the hierarchical structure of the codebook. Both theoretical analysis and extensive simulation results demonstrate that HBA can determine the optimal beam with high probability and short latency. Moreover, the hierarchical codebook design for indoor $\mathrm{THz}$ propagation is still an open problem. We adopt the hierarchical DFT codebook proposed in [9] to the LOS THz scenario. Furthermore, we propose a data-driven method according to [17] to design a hierarchical codebook utilizing hierarchical k- means clustering for the NLOS THz scenario.

This paper is organized as follows. The system model, channel model and beamforming framework are described in Section 2. In Section 3, the codebook design problems in both LOS and NLOS THz propagation are formulated. The hierarchical DFT codebook is proven to be a local optimum for the LOS codebook problem and is selected as the codebook for LOS HBA. A data-driven codebook design algorithm based on hierarchical k-means clustering is proposed for the NLOS HBA. The HBA algorithm for the SC-FDMA system operating in both LOS and NLOS scenarios is discussed in Section 4. Numerical results are provided in Section 5, followed by conclusions in Section 6.

Notation: Bold lowercase letters (e.g., a) and uppercase letters (e.g., A) represent column vectors and matrices, respectively. $A_{i, j}$ represents the $(i, j)$-th entry of A. $a_{k}$ and $\mathbf{a}_{k}$ stand for the $k$ th entry of $\mathbf{a}$ and the $k$ th column of A, respectively. $(\cdot)^{T},(\cdot)^{*},(\cdot)^{H}$ denote the transpose, conjugate and Hermitian transpose of a vector or matrix, respectively. All complex-valued gradients follow the definition according to [18].

\section{SYSTEM MODEL}

\subsection{System model}

The block diagram of the SU MIMO SC-FDMA transmission is shown in Fig. 1.

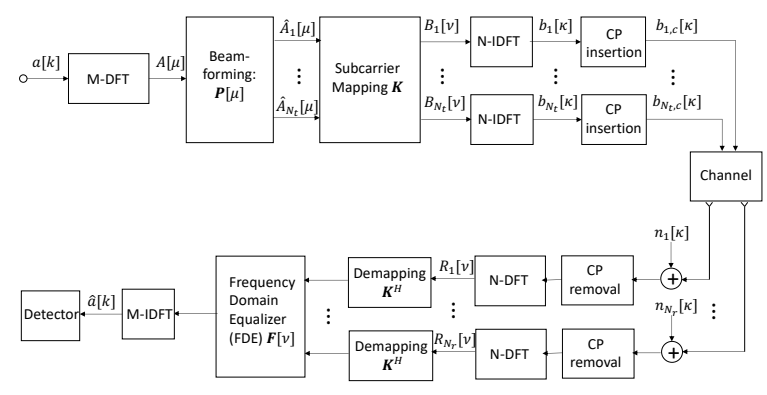

Fig. 1 - Structure of SU-MIMO SC-FDMA transmission system.

Here, the base station and the user terminal are equipped with $N_{t}$ and $N_{r}$ antennas, respectively, where $N_{t}$ is set to be a power of two, e.g., $N_{t}=4,8$, or 16 .Let $a_{s}[k], k \in$ $\{0,1, \cdots, M-1\}$ be the transmit data sequence with variance $\sigma_{a}^{2}$ corresponding to the symbol vector $\mathbf{a}_{s}$ for transmission in the SC-FDMA system. For simplicity, only a single block is considered in the following. An $M$-point DFT precoding is applied to symbol block $\mathbf{a}_{s}$ to transform $\mathbf{a}_{s}$ to an $M \times 1$ vector $\mathbf{A}=\mathbf{W}_{M} \mathbf{a}_{s}$ in the frequency domain with $\mathbf{A}=[A[0], A[1], \cdots, A[M-1]]^{T}$. Afterwards, an $N_{t} \times 1$ digital precoding vector $\mathbf{w}[\mu]$ is employed for each frequency domain symbol $A[\mu], 0 \leq \mu \leq M-1$ to form vectors of size $N_{t} \times 1$ via

$$
\hat{\mathbf{A}}[\mu]=\mathbf{w}[\mu] A[\mu]
$$

as shown in Fig. 1. Let $\hat{\mathbf{A}}_{i}=\left[\hat{A}_{i}[0], \hat{A}_{i}[1], \cdots, \hat{A}_{i}[M-1]\right]^{T}$ denote the $M \times 1$ pre-coded frequency domain vector cor- 
reponding to the $i$ th transmit antenna. $N_{t}$ vectors $\hat{\mathbf{A}}_{i}, i \in$ $\left\{1,2, \cdots, N_{t}\right\}$ are mapped to $N$ subcarriers , $N \geq M$, by the assignment matrix $\mathbf{K}$, leading to frequency domain vectors $\mathbf{B}_{i}$ with size $N$ which are represented as $\mathbf{B}_{i}=$ $\mathbf{K} \hat{\mathbf{A}}_{i}$. We assume the SC-FDMA subcarriers are assigned in a localized mode in this work, where symbols in $\hat{\mathbf{A}}_{i}$ are mapped to $M$ consecutive subcarriers starting from the $\nu_{0}$ th subcarrier, i.e., $\mathbf{K}=\left[\mathbf{0}_{\nu_{0} \times M}^{T} \mathbf{I}_{M}^{T} \mathbf{0}_{\left(N-M-\nu_{0}\right) \times M}^{T}\right]^{T}$. After performing the $N$-point inverse DFT operation on $\mathbf{B}_{i}$ and adding a Cyclic Prefix (CP) with a length of $L_{c}$, the time domain data symbols are transmitted.

At the receiver, first the $\mathrm{CP}$ is removed. Subsequently, the time-domain symbols are transformed to the frequency domain by an $N$-point DFT. The following matrix-vector model holds for the $\nu$ th subcarrier:

$$
\mathbf{R}[\nu]=\mathbf{H}[\nu] \mathbf{B}[\nu]+\mathbf{N}[\nu],
$$

where $\mathbf{R}[\nu], \mathbf{H}[\nu]$ and $\mathbf{N}[\nu]$ denote the received signal vector with size $N_{r} \times 1$, the $N_{r} \times N_{t}$ MIMO channel frequency response matrix and the noise vector at the $\nu$ th subcarrier, where $\mathbf{N}[\nu] \sim \mathcal{N}\left(\mathbf{0}, \sigma_{n}^{2} \mathbf{I}_{N_{T}}\right)$, respectively. Vector $\mathbf{B}[\nu]=\left[B_{1}[\nu], B_{2}[\nu], \cdots, B_{N_{t}}[\nu]\right]^{T}$ denotes the $N_{t} \times 1$ transmit signal vector at the $\nu$ th subcarrier. Since in the BA phase, the CSI is not known at Rx, the receiver is set to be quasi-omnidirectional. The average power $\bar{p}[\nu]$ of the received vector $\mathbf{R}[\nu]$ is given by

$$
\begin{aligned}
\bar{p}[\nu] & =\mathbb{E}\left\{\mathbf{R}^{H}[\nu] \mathbf{R}[\nu]\right\} \\
& =\mathbf{w}^{H}\left[\nu-\nu_{0}\right] \mathbf{H}^{H}[\nu] \mathbf{H}[\nu] \mathbf{w}\left[\nu-\nu_{0}\right] \sigma_{a}^{2}+N_{r} \times \sigma_{n}^{2} .
\end{aligned}
$$

The objective of the developed SC-FDMA beamforming is to maximize the average receive signal power $\bar{p}[\nu]$ for each subcarrier under a transmit power constraint. After the BA phase, the CSI can be acquired by the channel estimation approach proposed in [11] due to the large receive signal power after BA. Therefore, we focus on the BA task at the transmitter in this work.

\subsection{Channel model}

In this work, the indoor transmission scenario in Fig. 2 is considered. The corresponding room has dimensions $5 \mathrm{~m} \times 5 \mathrm{~m} \times 3 \mathrm{~m}$. The transmitter is fixed at the center of the room ceilling in order to cover the entire room, i.e., at position $(2.5 \mathrm{~m}, 2.5 \mathrm{~m}, 3 \mathrm{~m})$. Meanwhile, the receiver is placed uniformly within the given room. Representing a cellphone or a laptop, the receiver is positioned at a height of $1.5 \mathrm{~m}$. Both transmitter and receiver are equipped with a Uniform Linear Array(ULA) along the $y$-axis with antenna element spacing $r$. Indoor THz channel models have been developed in [8], [6],[19],[20]. In this paper, we mainly adopt the deterministic channel model based on the raytracing technique according to [6] and [21], since it describes the $\mathrm{THz}$ channel more accurately compared to the statistical channel models in [20] and [19].

Based on the sparsity of the given indoor scenario, the received Multipath Components (MPCs) typically comprise

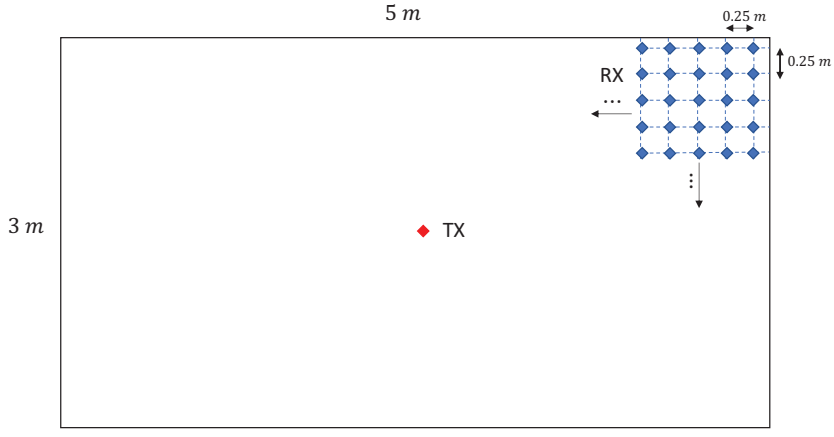

Fig. 2 - Indoor propagation scenario.

a Line-Of-Sight (LOS) component and several Non-LineOf-Sight (NLOS) components caused by reflection and scattering. Different MPCs have different Angles of Departure (AoDs) and Angles of Arrival (AoAs).The superposition of LOS channel and NLOS channel including transmit and receive filtering results in the discrete-frequency channel matrix $\mathbf{H}[\nu]$ for the $\nu$ th subcarrier. According to the channel modeling in [6], [22], and [23], the MIMO channel frequency response for the LOS component depends on the subcarrier frequency $f_{\nu}$ and the distance between transmitter and receiver $d$, and is denoted by $\mathbf{H}_{L O S}\left(f_{\nu}, d\right)$. The MIMO channel frequency response for the NLOS components is a function of the subcarrier frequency $f_{\nu}$ and the geometry information of the propagation scenario collected in vector $\zeta$, and is denoted by $\mathbf{H}_{N L O S}\left(f_{\nu}, \zeta\right)$. Thus, the MIMO channel frequency response for the LOS component $\mathbf{H}_{L O S}\left(f_{\nu}, d\right)$ and the NLOS components $\mathbf{H}_{N L O S}\left(f_{\nu}, \zeta\right)$, respectively, for the $\nu$ th subcarrier with frequency $f_{\nu}$ are given by

$$
\begin{aligned}
& \mathbf{H}_{L O S}\left(f_{\nu}, d\right)=\alpha_{L O S}\left(f_{\nu}, d\right) G_{t} G_{r} \\
& \mathbf{a}\left(N_{r}, \theta_{L O S}\right) \mathbf{a}\left(N_{t}, \phi_{L O S}\right)^{T} \\
& \mathbf{H}_{N L O S}\left(f_{\nu}, \zeta\right)= \sum_{i=1}^{n_{N L O S}} \alpha_{i}\left(f_{\nu}, \zeta_{i}\right) G_{t} G_{r} \\
& \times \mathbf{a}\left(N_{r}, \theta_{i}\left(\zeta_{i}\right)\right) \mathbf{a}\left(N_{t}, \phi_{i}\left(\zeta_{i}\right)\right)^{T} .
\end{aligned}
$$

Here, $\phi_{L O S}, \phi_{i}\left(\zeta_{i}\right), \theta_{L O S}$ and $\theta_{i}\left(\zeta_{i}\right)$ are the AoD of LOS component, AoDs of the corresponding NLOS components, AoA of LOS component and AoAs of the corresponding NLOS components, respectively. Since the reflection and scattering behaviors for all subcarriers are assumed to be the same, the AoAs and AoDs are modeled as constant over frequency. Their values can be computed accurately by the adopted ray-tracing algorithm based on the geometry information $\zeta_{i}, i \in\left\{1,2, \cdots, n_{N L O S}\right\}$ of the corresponding scattering scenario. Here, $\zeta_{i}$ contains the distance between transmitter and scattering point $d_{i, 1}$, the distance between scattering point and receiver $d_{i, 2}$, the incident angle $\theta_{i, 1}$, reflected angle $\theta_{i, 2}$ and the scattering direction $\theta_{i, 3}$. More details on the definition of the mentioned parameters and AoA/AoD calculation can be found in [6]. $G_{t}$ and $G_{r}$ are the transmit and receive antenna gains, respectively, and $\mathbf{a}\left(N_{t}, \theta\right)$ and $\mathbf{a}\left(N_{r}, \phi\right)$ denote the transmit and receive array steering vectors, 
which can be expressed as

$$
\mathbf{a}\left(N_{a}, \phi\right)=\left[1, \cdots, e^{j \frac{2 \pi f_{\nu} r}{c} n \sin \phi}, \cdots, e^{j \frac{2 \pi f_{\nu} r}{c}\left(N_{a}-1\right) \sin \phi}\right]^{T},
$$

where $n$ is the antenna element index with $0 \leq n \leq$ $N_{a}-1$. $N_{a}$ denotes the number of antennas, $r$ is the antenna spacing and $c$ is the speed of light. In an indoor THz channel, the LOS path gain $\left|\alpha_{L O S}\left(f_{\nu}, d\right)\right|^{2}$ depends on the spreading loss $L_{\text {spread }}\left(f_{\nu}, d\right)$ and absorption loss $L_{a b s}\left(f_{\nu}, d\right)$,

$$
\begin{aligned}
\left|\alpha_{L O S}\left(f_{\nu}, d\right)\right|^{2} & =L_{\text {spread }}\left(f_{\nu}, d\right) L_{a b s}\left(f_{\nu}, d\right), \\
L_{\text {spread }}\left(f_{\nu}, d\right) & =\left(\frac{c}{2 \pi f_{\nu} d}\right)^{2}, \\
L_{a b s}\left(f_{\nu}, d\right) & =e^{-k_{a b s}\left(f_{\nu} d\right)} .
\end{aligned}
$$

In (6), $d$ stands for the distance between transmitter and receiver, and $k_{a b s}\left(f_{\nu}, d\right)$ denotes the frequencydependent medium absorption coefficient. The NLOS components depend additionally on the reflection coefficient of the correponding surface. The squared magnitude of the $i$ th NLOS coefficient assuming first-order reflection of the reflecting surface is given by

$$
\begin{aligned}
\left|\alpha_{i}\left(f_{\nu}, \zeta_{i}\right)\right|^{2} & =\Gamma_{i}^{2}\left(f_{\nu}, \zeta_{i}\right)\left(\frac{c}{2 \pi f_{\nu}\left(d_{i, 1}+d_{i, 2}\right)}\right)^{2} \\
& \times e^{-k_{a b s}\left(f_{\nu}\left(d_{i, 1}+d_{i, 2}\right)\right)},
\end{aligned}
$$

where $\Gamma_{i}\left(f_{\nu}, \zeta_{i}\right)$ is the product of the Fresnel reflection coefficient and the scattering coefficient and can be calculated as given in [6]. Due to the high reflection loss in the $\mathrm{THz}$ band, the restriction to first-order reflection MPCs is justified.

In [22], the authors advocated the extension of a statistical channel model to the $\mathrm{THz}$ case in order to characterize the reflection behavior of the furniture. As discussed in [8], [24] and [22], modeling the reflection components due to furniture by the ray-tracing technique is not feasible due to an extremely high computational load. Thus, we adopt the hybrid channel modeling idea in [22] that the LOS component and the reflection components due to the walls and ceiling are generated by the ray-tracing technique and the reflection components due to the furniture are generated via the Saleh-Valenzuela (S-V) channel model. We adopt the parameters' setting of the S-V channel model in [22]. The details of this hybrid channel modeling can be found in [22].

\subsection{Beamforming framework}

From a system level, the beamforming at the transmitter is required to maximize the sum receive power over the transmission band. If $\mathbf{H}[\nu]$ for all $\nu=1,2, \cdots, N$ is known at the transmitter, the optimal beamforming vector can be computed using the approach provided in [10]. However, in THz communications it is not practical to perform an entry-wise estimation of the channel matrix at the transmitter, which has a large scale due to the large antenna arrays. Thus, selecting the beam code from a predefined codebook is frequently considered in THz communications.

For a LOS scenario in THz band, the channel is dominated by the LOS component assuming its power is significantly larger than that of the other MPCs [8], [6]. In such a case, the beamforming problem is simplified to finding the AoD of the LOS component, and setting the beamforming vector as the steering vector pointing to the AoD of the LOS component. Thus, the codebook is composed only of steering vectors. In addition, in the LOS scenario, the coherence bandwidth is relatively larger due to the dominance of the LOS component, i.e., the LOS channel exhibits a flatter frequency response than that of the NLOS channel. Thus, neighboring subcarriers can employ the same beamforming vector.

However, in an indoor THz scenario, the LOS propagation might be blocked by the presence of obstacles like moving persons, furniture, or many diverse objects [6]. In that case, the codebook cannot be designed as the composition of steering vectors anymore but has to be specifically tailored to the NLOS scenario. Also, the NLOS channel may be frequency-selective due to the absence of the LOS component. Therefore, each subcarrier should be assigned a carefully designed code word.

According to the aforementioned difference between the LOS beamforming and the NLOS beamforming, a prejudgement regarding the propagation scenario has to be conducted before beamforming. Since the LOS component power is nearly $20 \mathrm{~dB}$ larger than that of the NLOS components in the considered typical indoor scenario, the channel can be classified by the receive power. For a training block, if the measured receive power is higher than a threshold $P_{t h}$, the channel will be classified to a LOS channel, and the LOS beamforming procedure will be performed in the BA phase. Otherwise, the channel will be considered as a NLOS channel and the procedure for the NLOS beamforming will take place in the following BA phase. The power threshold $P_{t h}$ depends on the geometry of the propagation scenario, the humidity of the atmosphere and the material of the reflecting objects. Thus, the power threshold must be designed based on the given propagation scenario and no general rule regarding its selection can be introduced. The adopted power threshold choice will be discussed in detail in Section 3. After the channel assessment, the beamforming vectors for each subcarrier are determined by the corresponding BA procedure, which will be discussed in detail in Section 4.

\section{CODEBOOK DESIGN}

In this section, we discuss the hierarchical beam codebook used in hierarchical beam alignment. Hierarchical codebooks have been already studied in [25]. The hierarchical codebook is also the core of the hierarchical beam alignment, which helps to improve the efficiency in searching for the best beam code. In this section, we first introduce the definition of the hierarchical codebooks, 


\begin{tabular}{|c|c|c|c|c|c|c|c|c|}
\hline \multirow{3}{*}{$\begin{array}{l}\text { The first layer } \\
\text { The second layer }\end{array}$} & \multicolumn{8}{|c|}{ Channel Domain $\mathcal{H}$} \\
\hline & \multicolumn{8}{|c|}{$\boldsymbol{w}(0,1)$} \\
\hline & \multicolumn{4}{|c|}{$\boldsymbol{w}(1,1)$} & \multicolumn{4}{|c|}{$\boldsymbol{w}(1,2)$} \\
\hline The third layer & \multicolumn{2}{|c|}{$\boldsymbol{w}(2,1)$} & \multicolumn{2}{|c|}{$\boldsymbol{w}(2,2)$} & \multicolumn{2}{|c|}{$\boldsymbol{w}(2,3)$} & \multicolumn{2}{|c|}{$\boldsymbol{w}(2,4)$} \\
\hline The last layer & $\boldsymbol{w}(3,1)$ & $\boldsymbol{w}(3,2)$ & $\boldsymbol{w}(3,3)$ & $\boldsymbol{w}(3,4)$ & $\boldsymbol{w}(3,5)$ & $w(3,6)$ & $\boldsymbol{w}(3,7)$ & $\boldsymbol{w}(3,8)$ \\
\hline
\end{tabular}

Fig. 3 - The beam coverage of a 3-layer hierarchical codebook.

then discuss the design of the hierarchical codebooks in the LOS scenarios and the NLOS scenarios.

\subsection{Definition}

Before introducing the definition of a hierarchical codebook, we first define the beam coverage $C V\left(\mathbf{w}_{i}\right)$ of a code word $\mathbf{w}_{i}$ within a codebook $\mathbf{W}$. Let $\mathcal{H}$ denote the set of all possible channel snapshots within the given indoor propagation scenario, which is called the channel domain in the following. The beam coverage $C V\left(\mathbf{w}_{i}\right)$ of a code word $\mathbf{w}$ in a codebook $\mathbf{W}$ is a subset of the channel domain $\mathcal{H}$, where the receive power with $C V\left(\mathbf{w}_{i}\right)$ is strongest among all code words in $\mathbf{W}$, i.e.,

$$
C V\left(\mathbf{w}_{i}\right)=\left\{\mathbf{H} \mid\left\|\mathbf{H} \mathbf{w}_{i}\right\|_{2}=\max _{\mathbf{w}_{j} \in \mathbf{W}}\left\|\mathbf{H} \mathbf{w}_{j}\right\|_{2}, \mathbf{H} \in \mathcal{H}\right\} .
$$

Note that for each channel realization $\mathbf{H}$ in $\mathcal{H}$, there is always a code word in $\mathbf{W}$ aligned to $\mathbf{H}$. In other words, the union of the beam coverages of all code words composes the channel domain $\mathcal{H}$, i.e.,

$$
\cup_{\mathbf{w}_{j} \in \mathbf{W}} C V\left(\mathbf{w}_{j}\right)=\mathcal{H} .
$$

A hierarchical codebook is a family of $I$ sub-codebooks $\mathbf{W}^{i}, 1 \leq i \leq I$. In this work, we only consider the binary hierarchical codebook, which means the size of the $i$ th codebook is twice of the size of the $(i-1)$ th codebook. The code words in the sub-codebook are layered due to their beam coverage as shown in Fig. 3. Let $\mathbf{w}(i, j), 1 \leq$ $i \leq I, 1 \leq j \leq 2^{i-1}$ denote the $j$ th code word in the $i$ th layer. The beam coverage of all code words in the first $I-1$ layers must satisfy the following criterion:

Criterion 1: The beam coverage $C V(\mathbf{w}(i, j))$ of an arbitary code word $\mathbf{w}(i, j)$ should be the union of those of two code words in the next layer, i.e.,

$C V(\mathbf{w}(i, j))=C V(\mathbf{w}(i+1,2 j-1)) \cup C V(\mathbf{w}(i+1,2 j))$,

where $0 \leq i \leq I-1 . \mathbf{w}(i, j)$ is called the parent of $\mathbf{w}(i+$ $1,2 j-1)$ and $\mathbf{w}(i+1,2 j)$, and $\mathbf{w}(i+1,2 j-1), \mathbf{w}(i+1,2 j)$ are called the children of $\mathbf{w}(i, j)$.

The above criterion guarantees the codebook is tree-like, which can be used in the hierarchical beam alignment. In the HBA algorithm, which will be introduced in Section 4, the algorithm converges to a beam code in the highest layer with high probability. Thus, the average beamforming gain of a given hierarchical codebook $\mathbf{W}=$ $\left\{\mathbf{W}^{1}, \mathbf{W}^{2}, \cdots, \mathbf{W}^{I}\right\}$ is defined as the average receive power $\bar{r}(\mathbf{W})$ of the best beam code in the highest layer, i.e.,

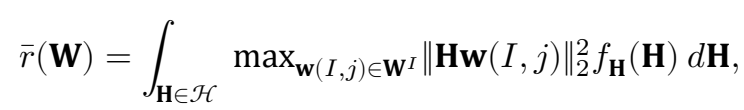

where $f_{\mathbf{H}}(\mathbf{H})$ is the Probability Density Function (PDF) of $\mathbf{H}$. The aim of the codebook design is to generate a codebook $\mathbf{W}$ satisfying Criterion 1 meanwhile maximizing the beamforming gain $\bar{r}(\mathbf{W})$ over $\mathcal{H}$. Meanwhile, the transmit power must be limited. Without loss of generality, we assume that the $j$-th beamforming code word $\mathbf{w}(i, j)$ in the $i$-th layer has unit norm, i.e., $\mathbf{w}^{H}(i, j) \mathbf{w}(i, j)=1$.

The codebook design problem introduced above is formulated on the basis of the codebook design in [25]. However, in [25], mainly the criteria for a hierarchical codebook are proposed and the objective function of the codebook design problem is not explicitly stated. In this work, the two criteria for the hierarchical codebook in [25] are modified to a single criterion by adjusting the definition of the beam coverage. In addition, the beamforming gain of the codes in a hierarchical codebook is utilized in this work as an objective function, which can be regarded as a supplement of the codebook design in [25].

\subsection{LoS codebook design}

In this subsection, a LoS propagation scenario is considered, where the LoS component is always obtained. Based on the measurement results in [20], the power of the LOS component is nearly $20 \mathrm{~dB}$ larger than the power of the NLOS components in the $\mathrm{THz}$ band in a typical propagation scenario due to the high reflection and scattering loss. Therefore, the channel in THz LOS scenario is in principle LOS-dominant. In this case, the beam should be focused on the LOS component.

In this paper, the receiver position is assumed to be uniformly distributed within the considered indoor environment in Fig. 2. Thus, the LOS spatial angle $\Phi=\frac{2 \pi f_{\nu} r}{c} \sin \phi_{L O S}$ is considered as uniformly distributed within $(0,2 \pi)$, where $r$ is the antenna spacing. Due to the LOS-dominant channel property in the considered scenario, the beamforming gain of a given hierarchical codebook $\mathbf{W}=\left\{\mathbf{W}^{1}, \mathbf{W}^{2}, \ldots, \mathbf{W}^{I}\right\}$ is defined as the the receive power of the best beam code in the highest layer for the LOS component with spatial angle $\Phi$, i.e.,

$$
g(\Phi)=\max _{\mathbf{w} \in \mathbf{W}^{I}}\left\|\mathbf{a}_{s}^{T}\left(N_{T}, \Phi\right) \mathbf{w}\right\|_{2}^{2} .
$$

where $\mathbf{a}_{s}\left(N_{T}, \Phi\right)=\left[1, \cdots, e^{j \Phi}, \cdots, e^{j\left(N_{T}-1\right) \Phi}\right]^{T}$. The codebook design problem is equivalent to maximizing the average beamforming gain over $\Phi \in(0,2 \pi)$. Hence, the 

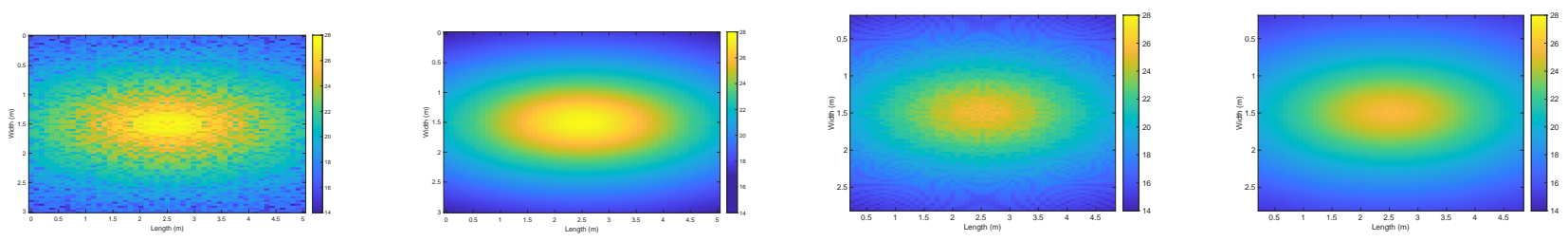

(a) Beamforming gain in $\mathrm{dB}$ of the hi- (b) Beamforming gain in $\mathrm{dB}$ of the (c) Beamforming gain in $\mathrm{dB}$ of the (d) Beamforming gain in $\mathrm{dB}$ of the erarchical DFT codebook in the LOS maximum ratio transmission in the hierarchical DFT codebook in the maximum ratio transmission in the indoor scenario without furniture. LOS indoor scenario without furni- LOS indoor scenario with furniture. LOS indoor scenario with furniture. ture.

Fig. 4 - Beamforming gain of different codebooks in the given indoor propagation scenario with a carrier frequency of 100 GHz single-frequency transmission.

codebook design problem can be written as

$$
\begin{aligned}
\max _{\mathbf{W}} & \int_{\Phi=0}^{2 \pi} g(\Phi) d \Phi \\
\text { s.t. } & \mathbf{w}^{H}(i, j) \mathbf{w}(i, j)=1,1 \leq i \leq I, 1 \leq j \leq 2^{i-1} \\
& C V(\mathbf{w}(i, j))=C V(\mathbf{w}(i+1,2 j-1)) \cup \\
& C V(\mathbf{w}(i+1,2 j)), 1 \leq i \leq I-1,1 \leq j \leq 2^{i-1},
\end{aligned}
$$

where $\mathbf{w}(i, j)$ denotes the $j$ th code word in the $i$ th layer. The above optimization problem is a non-convex problem due to the non-convex constraints. Hence, it is difficult to obtain the globally optimum solution for the codebook $\mathbf{W}$. However, the objective function value depends only on the sub-codebook in the highest layer, i.e., $\mathbf{W}^{I}=$ $\left[\mathbf{w}_{1}, \mathbf{w}_{2}, \cdots, \mathbf{w}_{2^{I-1}}^{T}\right]$. One greedy approach for the codebook design problem is to choose the sub-codebook in the highest layer $\mathbf{W}^{I}$ for maximization of the objective function value. Then, the highest sub-codebook $\mathbf{W}^{I}$ is extended to a hierarchical codebook. The design problem for the highest layer sub-codebook $\mathbf{W}^{I}$ is given by

$$
\begin{aligned}
\max _{\mathbf{W}^{I}} & \int_{\phi} \max _{\mathbf{w} \in \mathbf{W}^{I}}\left\|\mathbf{a}^{T}\left(N_{T}, \phi\right) \mathbf{w}\right\|_{2}^{2} d \phi \\
\text { s.t. } & \mathbf{w}^{H}(I, j) \mathbf{w}(I, j)=1,1 \leq j \leq 2^{I-1} .
\end{aligned}
$$

This optimization problem is non-convex since the objective function is a non-concave function. Theorem 1 shows that the DFT codebook will be one of the locally optimum solutions for the above optimization problem if the highest layer sub-codebook size is fixed to $N_{T}$. In [9], the unitary DFT matrix $\mathbf{W}_{N_{t}}$ is chosen as the codebook for the beam alignment, which is given by

$\mathbf{W}_{N_{t}}=\frac{1}{\sqrt{N_{t}}}\left[\mathbf{a}_{s}\left(N_{t}, \Phi_{1}\right), \cdots, \mathbf{a}_{s}\left(N_{t}, \Phi_{n}\right), \cdots, \mathbf{a}_{s}\left(N_{t}, \Phi_{N_{t}}\right)\right]$,

with $\mathbf{a}_{s}\left(N_{t}, \Phi_{n}\right)=\left[1, e^{j \Phi_{n}}, \cdots, e^{j\left(N_{t}-1\right) \Phi_{n}}\right]^{T}$. Here, $\Phi_{n}=$ $\frac{\pi(2 n-1)}{N_{t}}, n \in\left\{1, \cdots, N_{t}\right\}$ denotes the spatial angle of the $n$-th beam.

Theorem 1: The unitary DFT matrix $\mathbf{F}$ is one of the locally optimum solutions for the above optimization problem if the codebook size is fixed to $N_{T}$

\section{Proof. See Appendix A.}

Fig. 4 illustrates the beamforming gain for the LOS case in the given indoor scenario. Here, $100 \times 100$ sampling points are considered and their beamforming gains are obtained. The beamforming gain definition can be found in (11), which is the objective function of the proposed codebook design. The performance of the resulting codebook can be evaluated by the beamforming gain shown in Fig. 4.

In Fig. 4(a) and 4(b), only the multipath component generated by a ray-tracing algorithm is considered. Fig. 4(a) illustrates the beamforming gain of the DFT codebook in the considered LOS indoor propagation scenario. Compared to the beamforming gain of the Maximum Ratio Transmission (MRT) in Fig. 4(b), which is considered as the upper bound of the beamforming gain, the beamforming gain of the DFT codebook within the entire indoor environment is close to that of MRT. Hence, the hierarchical DFT codebook entails only a slight performance loss with a small codebook size, which can be considered as a welltailored codebook for the LOS scenario.

Fig. 4(c) and Fig. 4(d) illustrate the beamforming gain of the DFT codebook and MRT, respectively, for the LOS case in the given indoor scenario with furniture. The beamforming gains of $100 \times 100$ sampling points have been obtained. For one sampling point, 100 statistical channel snapshots have been generated to obtain the average beamforming gain. The difference between the beamforming gain of the DFT codebook and MRT is insignificant, which suggests that the DFT codebook achieves a promising performance even in a complex indoor scenario.

To generate the sub-codebooks in lower layers, the beam coverage of the DFT codebook should be determined. Based on the definition of the beam coverage in Criterion 1 , the beam coverage of a beam code $\mathbf{w}(I, j)$ in $I \mathrm{t}$ h layer is given by

$$
C V(\mathbf{w}(I, j))=\left(\frac{2 \pi(j-1)}{N_{T}}, \frac{2 \pi j}{N_{T}}\right) .
$$


According to Criterion 1 of the hierarchical codebook, the beam coverage of a beam code $\mathbf{w}(i, j), i=1,2, \cdots, I-1$ is determined by

$$
C V(\mathbf{w}(i, j))=\left(\frac{2 \pi(j-1) 2^{I-i}}{N_{T}}, \frac{2 \pi j 2^{I-i}}{N_{T}}\right) .
$$

In the LOS scenario, a code word $\mathbf{w}(i, j)$ is identical to the steering vector located in the center of its corresponding beam coverage $C V(\mathbf{w}(i, j))$, which is given by

$$
\mathbf{w}(i, j)=\mathbf{a}\left(N_{T}, \Phi_{i, j}\right)
$$

where $\Phi_{i, j}=\frac{\pi(2 j-1) 2^{I-i}}{N_{T}}$. Theorem 1: The beam coverage $C V(\mathbf{w}(i, j))$ of the $j$-th code word in the $i$-th layer can be expressed as

$$
C V(\mathbf{w}(i, j))=\left\{\Phi \mid \Phi \in\left[\frac{2 \pi(2 j-2)}{2^{i}}, \frac{4 j \pi}{2^{i}}\right]\right\} .
$$

Proof. See Appendix B.

\subsection{NLOS codebook design}

In an indoor scenario, the LOS component might be blocked by obstacles such as persons, furniture, or many other diverse objects. In this case, utilizing the power from NLOS components will be essential for the beamforming performance. Consider a hierarchical codebook $\mathbf{W}=\left\{\mathbf{W}^{1}, \mathbf{W}^{2}, \cdots, \mathbf{W}^{I}\right\}$ with $I$ layers. The codebook design problem can be written as

$$
\begin{aligned}
\max _{\mathbf{W}} & \int_{\zeta} \max _{\mathbf{w}(I, j) \in \mathbf{W}^{I}}\left\|\mathbf{w}(I, j) \mathbf{H}_{N L O S}\left(f_{\nu}, \zeta\right)\right\|_{2}^{2} \\
& f_{\zeta}(\zeta) \mathrm{d} \zeta \\
\text { s.t. } & \mathbf{w}^{H}(i, j) \mathbf{w}(i, j)=1, \\
& i=1,2, \cdots, I, j=1,2, \cdots, 2^{i-1} \\
& C V(\mathbf{w}(i, j)) \\
& =C V(\mathbf{w}(i+1,2 j-1)) \cup C V(\mathbf{w}(i+1,2 j))
\end{aligned}
$$

where $f_{\zeta}(\zeta)$ is the PDF of the geometry information. However, modeling the NLOS components' behavior in the $\mathrm{THz}$ band is still an open problem. It is difficult to model the NLOS channel analytically including the reflection and scattering behavior. Faced with this challenge in the NLOS scenario, it is generally intricate to find an analytical method to generate the codebook in the NLOS scenario. Therefore, a data-driven codebook design such as the one proposed in [17] is of great benefit for the considered indoor THz NLOS scenario. In the data-driven approach, the integral over the indoor scenario is approximated by the average over $N_{p}$ realizations of the indoor channel. In particular, the objective function is defined as

$$
f_{N L O S}(\mathbf{W})=\sum_{i=1}^{N_{p}} \max _{\mathbf{w}(I, j) \in \mathbf{W}^{I}}\left\|\mathbf{H}_{N L O S}^{\nu, i} \mathbf{W}(I, j)\right\|_{2}^{2},
$$

where $\mathbf{H}_{N L O S}^{\nu, i}$ is the NLOS channel frequency response of $i$ t $\mathrm{h}$ realization at frequency $f_{\nu}$. The corresponding modified codebook design problem reads

$$
\begin{array}{cl}
\max _{\mathbf{W}} & f_{N L O S}(\mathbf{W}) \\
\text { s.t. } & \mathbf{w}^{H}(i, j) \mathbf{w}(i, j)=1, \\
& i=1,2, \cdots, I, j=1,2, \cdots, 2^{i-1} \\
& C V(\mathbf{w}(i, j))= \\
& C V(\mathbf{w}(i+1,2 j-1)) \cup C V(\mathbf{w}(i+1,2 j)) .
\end{array}
$$

The above optimization problem cannot be solved directly since the second hierarchical structure constraint is non-convex. Hence, before solving the overall hierarchical codebook design problem, a single layer codebook design problem is solved since the objective function depends only on the highest layer sub-codebook. For a single layer codebook $\mathbf{W}=\left[\mathbf{w}_{1}, \mathbf{w}_{2}, \cdots, \mathbf{w}_{J}\right]$, where $J$ is the codebook size, the optimization problem is given by

$$
\begin{aligned}
\max _{\mathbf{W}} & \sum_{i=1}^{N_{p}} \max _{\mathbf{w}_{j} \in \mathbf{W}}\left\|\mathbf{H}_{N L O S}^{\nu, i} \mathbf{w}_{j}\right\|_{2}^{2} \\
\text { s.t. } & \mathbf{w}_{j}^{H} \mathbf{w}_{j}=1, j=1,2, \cdots, J
\end{aligned}
$$

Theorem 3: Let $\hat{\mathbf{H}}_{\nu, i}$ and denote $\left(\mathbf{H}_{N L O S}^{\nu, i}\right)^{H} \mathbf{H}_{N L O S}^{\nu, i}$. The distance $D(\mathbf{X}, \mathbf{Y})$ between two $N_{t} \times N_{t}$ matrices $\mathbf{X}$ and $\mathbf{Y}$ is defined as

$$
D(\mathbf{X}, \mathbf{Y})=\operatorname{tr}\left((\mathbf{X}-\mathbf{Y})(\mathbf{X}-\mathbf{Y})^{H}\right)
$$

The optimization problem in (22) is equivalent to a distance minimization problem, i.e.,

$$
\begin{aligned}
\min _{\mathbf{w}} & \sum_{i=1}^{N_{p}} \sum_{\nu=\nu_{0}}^{\nu_{0}+M-1} \min _{\mathbf{w}_{j} \in \mathbf{w}} D\left(\hat{\mathbf{H}}_{\nu, i}, \mathbf{w}_{j} \mathbf{w}_{j}^{H}\right) \\
\text { s.t. } & \mathbf{w}_{j}^{H} \mathbf{w}_{j}=1, j=1,2, \cdots, J
\end{aligned}
$$

Proof. See Appendix C.

One well-known algorithm to solve the distance minimization problem is k-means clustering, which is an unsupervised machine learning algorithm. Here $\hat{\mathbf{W}}_{j}, j=$ $1,2, \cdots, J$ and $\hat{\mathbf{H}}_{\nu, i}, i=1,2, \cdots, N_{p}$ are regarded as the clustering centers in k-means clustering and the training data set, respectively. The overall training data set is denoted as $\mathcal{H}_{N L O S}$. The algorithm proceeds by alternating between assignment step and update step as described in the following. 


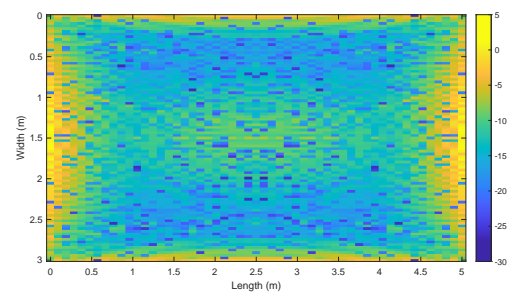

(a) Beamforming gain in $\mathrm{dB}$ of the hierarchical DFT (b) codebook in the NLOS case without furniture.

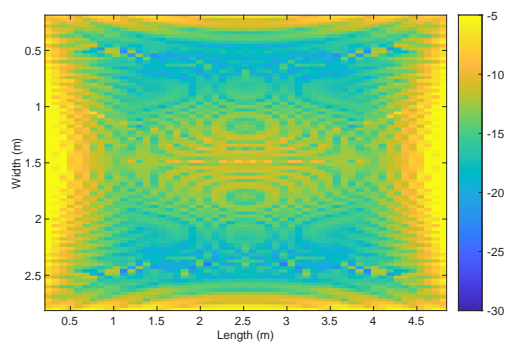

(d) Beamforming gain in $\mathrm{dB}$ of the hierarchical DFT codebook in the NLOS case with furniture.
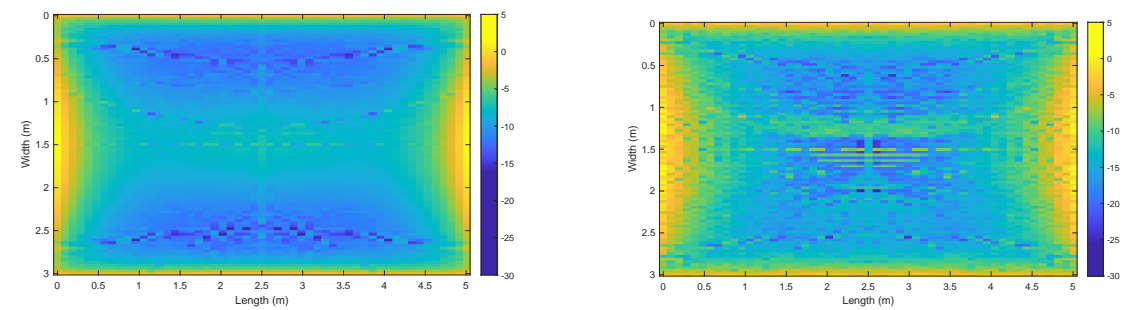

(b) Beamforming gain in $\mathrm{dB}$ of the maximum ratio (c) Beamforming gain in $\mathrm{dB}$ of the hierarchical ktransmission in the NLOS case without furniture. means codebook in the NLOS case without furniture.
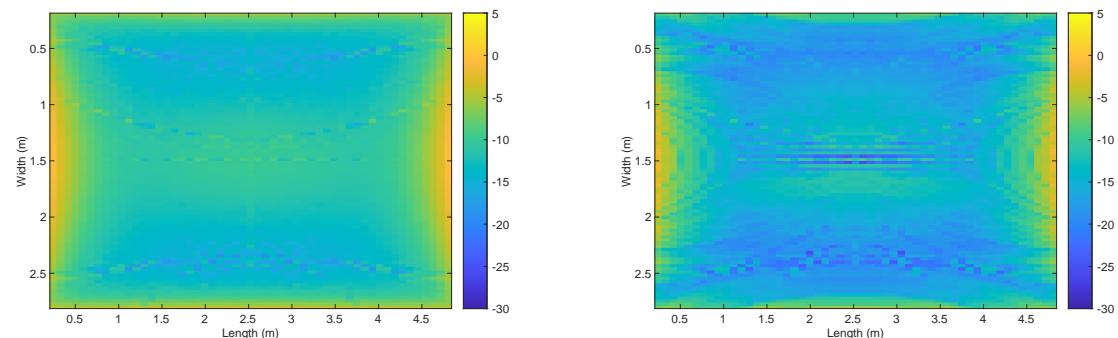

(e) Beamforming gain in $\mathrm{dB}$ of the maximum ratio (f) Beamforming gain in $\mathrm{dB}$ of the hierarchical $\mathrm{k}$ transmission in the NLOS case with furniture.

Fig. 5 - Beamforming gain of different approaches for the given indoor NLOS propagation scenario with carrier frequency of 100 GHz single-frequency transmission.

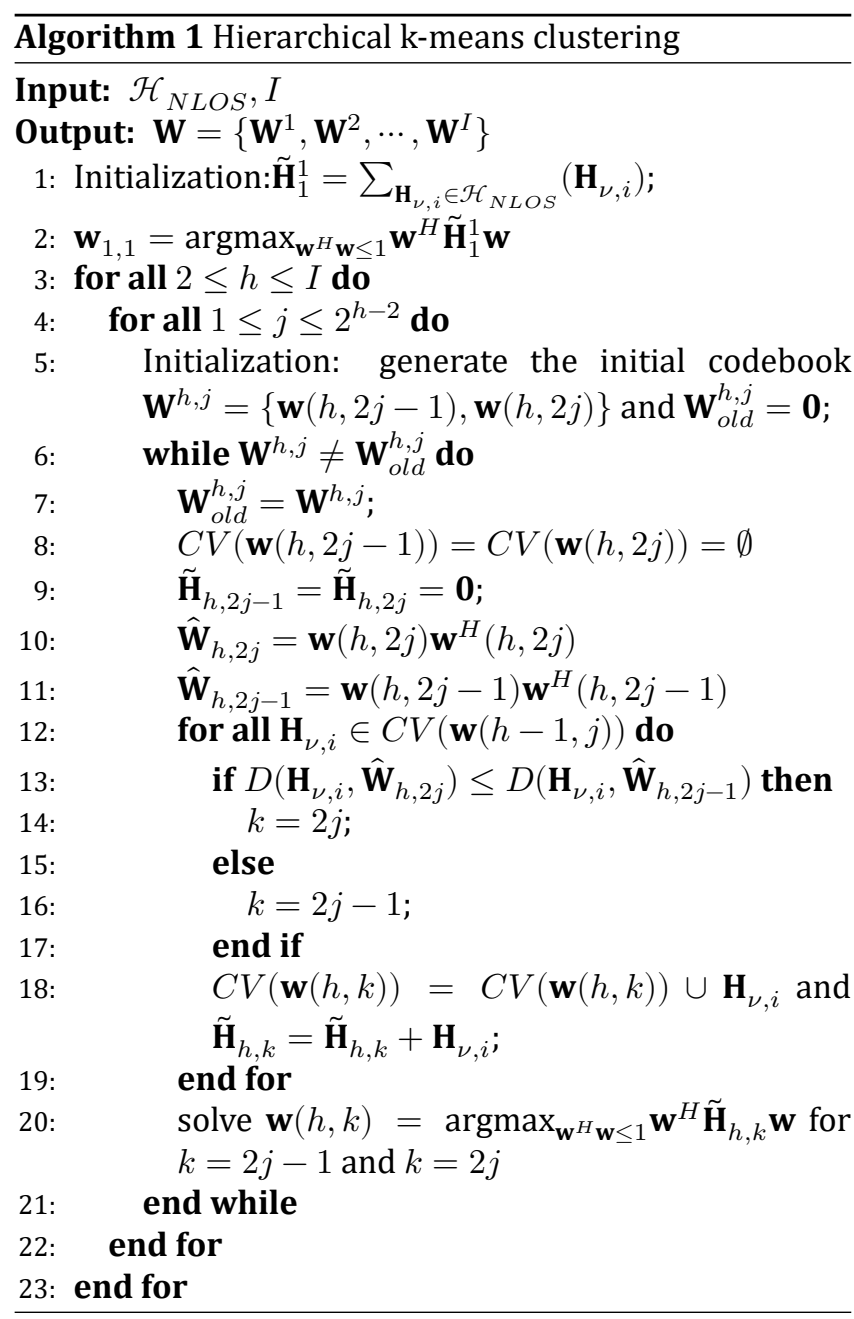

Assignment step: Assign each training channel $\hat{\mathbf{H}}_{i}$ to the corresponding clustering center $\hat{\mathbf{W}}_{j}$, which provides the minimum distance $D\left(\hat{\mathbf{H}}_{i}, \hat{\mathbf{W}}_{j}\right)$. This means the training channel set is divided into $J$ clusters, i.e., $\mathcal{H}_{1}, \mathcal{H}_{2}, \cdots, \mathcal{H}_{J}$. The $j$ th cluster is expressed mathmatically as

$$
\mathcal{H}_{j}=\left\{\hat{\mathbf{H}}_{\nu, i} \mid j=\operatorname{argmin}_{1 \leq j \leq J} D\left(\hat{\mathbf{H}}_{\nu, i}, \hat{\mathbf{W}}_{j}\right)\right\}
$$

Update step: Recalculate centers for the training channels assigned to each cluster. This is done by solving the following optimization problem for $j=1,2, \cdots, J$

$$
\begin{array}{cl}
\min _{\mathbf{W}} & \sum_{\mathbf{H} \in \mathcal{H}_{j}} D(\mathbf{H}, \mathbf{W}) \\
\text { s.t. } & \operatorname{tr}(\mathbf{W})=1, j=1,2, \cdots, J \\
& \operatorname{rank}(\mathbf{W})=1 .
\end{array}
$$

Theorem 4: The globally optimal solution of $\mathbf{W}$ for (26) is given by

$$
\mathbf{W}=\mathbf{w}_{\max } \mathbf{w}_{\text {max }}^{H},
$$

where $\mathbf{w}_{\text {max }}$ is the eigenvector of $\sum_{\mathbf{H} \in \mathcal{H}_{j}} \mathbf{H}$ corresponding to its largest eigenvalue.

Proof. See Appendix D.

However, the aforementioned approach only generates a single layer codebook, which cannot be adopted for HBA. To guarantee the hierarchical structure of the final resul-ting codebook, one variant of k-means clustering, named hierarchical k-means clustering, is introduced here. The procedure of hierarchical k-means clustering is shown in Algorithm 1. The most important property of hierarchical 
$\mathrm{k}$-means clustering is that it guarantees the hierarchical structure of the resulting codebook. Moreover, the hierarchical k-means algorithm can speed up k-means-based learning approaches. In the hierarchical k-means algorithm the branching factor is chosen to 2 , defining the number of clusters at each layer of the codebook. In the beginning, the beam coverage of $\mathbf{w}(1,1)$ is set to $\mathcal{H}_{N L O S}$. $\mathbf{w}(1,1)$ is selected as the eigenvector belonging to the largest eigenvalue of $\tilde{\mathbf{H}}_{1}^{1}=\sum_{\mathbf{H}_{\nu, i} \in \mathcal{H}_{N L O S}}\left(\mathbf{H}_{\nu, i}\right)$. Then, $\mathcal{H}_{\text {NLOS }}$ is clustered into two sub-clusters $C V(\mathbf{w}(2,1))$ and $C V(\mathbf{w}(2,1))$ by k-means clustering. We recursively further cluster each sub-cluster until we reach the required depth $H$ of the codebook.

Fig. 5 shows the beamforming gain for the NLOS case in the given indoor scenario. Again, in Fig. 5(c), 5(c) and 5 (b) only the multipath components generated by the raytracing algorithm are considered to obtain the beamforming gain profile. The beamforming gain of the hierarchical k-means codebook generated by the hierarchical k-means clustering is depicted in Fig. 5(c). Compared to the beamforming gain of the hierarchical DFT codebook and that of the MRT in Fig. 5(a) and Fig. 5(b), respectively, the hierarchical k-means codebook achieves a beamforming gain closer to that of the MRT than that of the DFT codebook. Hence, the designed hierachical codebook is a better choice than the DFT codebook in the considered NLOS propagation scenario.

Figures 5(e), 5(a) and 5(f) illustrate the beamforming gain of MRT, the DFT codebook and the hierarchical kmeans codebook, respectively, for the NLOS case in the indoor scenario with furniture. Again, $100 \times 100$ sampling points have been simulated and 100 statistical channel snapshots have been generated for each sampling point to obtain the average beamforming gain. Based on figures 5(e), 5(a) and 5(f), it can be concluded that in the indoor scenario with furniture, the proposed K-means codebook outperforms the DFT codebook.

From Fig. 5, it can be concluded that the maximal beamforming gain in the NLOS scenario is $5 \mathrm{~dB}$. Meanwhile, the minimal beamforming gain of the proposed algorithm in the LOS case is $15 \mathrm{~dB}$. Thus, a value of $10 \mathrm{~dB}$ is selected for this work as the power threshold $P_{t h}$ to judge whether the channel is a LOS channel or a NLOS channel.

\section{BEAM ALIGNMENT ALGORITHM}

In this section, we first formulate the beam alignment problems for both the LOS and NLOS scenarios, respectively. Next, the HBA algorithm is adopted to the considered SU-MIMO SC-FDMA system in both the LOS and NLOS scenarios to identify the optimal beam.

\subsection{Problem formulation}

BA is a process to identify the optimal beam code from a predefined codebook, which guarantees maximal received signal power. Since the channel behavior under the LOS and the NLOS scenarios differs significantly in the

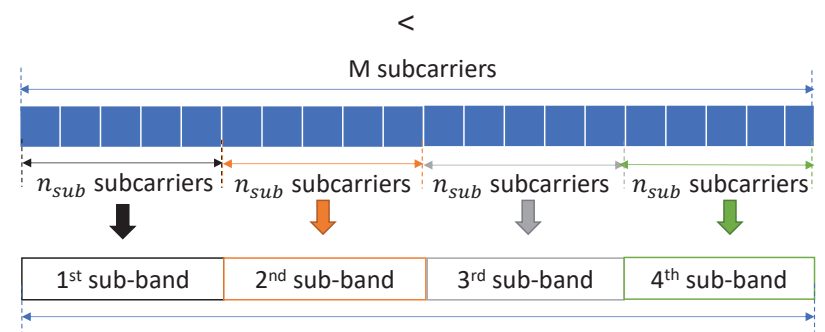

$n_{s}$ sub-bands

Fig. 6 - sub-band assignment for SC-FDMA system.

THz band, the BA problems are formulated for both cases separately in the following.

\subsubsection{LOS beam alignment}

In the LOS scenario, the channel behavior is dominated by the LOS propagation [23]. Hence, the beam codebook is selected as the hierarchical DFT codebook discussed in Section 3. The precoded signal at the SC-FDMA transmitter should be steered into the direction of the AoD of the LOS component $\phi_{L O S}$. The correponding spatial angle of the channel is given by $\Phi=\frac{2 \pi f_{c} r}{c} \sin \phi_{L O S}$, where $f_{c}$ is the carrier frequency of the transmission, $r$ is the antenna spacing and $c$ is the speed of light. For a transmission bandwidth $B W$, the maximal variation of the LOS spatial angle over the transmission bandwidth $\Delta \Phi$ is given by $\Delta \Phi=\frac{2 \pi B W r}{c} \sin \phi_{L O S}$. For a broadband SC-FDMA system, if the same beam code is assigned to all subcarriers, some of the subcarriers may not be aligned accurately. To cope with the beam misalignment caused by a large bandwidth, all available subcarriers are divided into $n_{s}$ subbands as shown in Fig. 6, where $n_{\text {sub }}$ is the size of one subband. Here, the same beam code is assigned to all subcarriers within the same sub-band. Therefore, the maximal spatial angle variation within a sub-band $\Delta \Phi_{\text {sub }}$ should not exceed the beam coverage of the code word in the highest layer sub-codebook $\mathbf{W}^{I}$, i.e., $\max _{\phi_{L O S}} \Delta \Phi_{\text {sub }} \leq$ $\frac{2 \pi}{N_{T}}$. Hence, $n_{s}$ can be determined by

$$
\begin{aligned}
\max _{\phi_{L O S}} \Delta \Phi_{s u b} & \leq \frac{2 \pi}{N_{T}} \\
\frac{2 \pi B W r}{c n_{s}} & \leq \frac{2 \pi}{N_{T}} \\
n_{s} & \geq \frac{B W r N_{T}}{c}
\end{aligned}
$$

The BA problem is equivalent to finding the optimal beam code $\mathbf{w}_{i}^{*}$ from $\mathbf{W}_{D F T}$ for the $i$ th sub-band maximizing the mean received power over the $i$ th sub-band. The mean received power $P_{i}\left(\mathbf{w}_{i}\right)$ for precoding vector $\mathbf{w}_{i}$ for $i$ th subband is defined as

$$
P_{i}\left(\mathbf{w}_{i}\right)=\sum_{\nu=\nu_{0}(i)}^{\nu_{0}(i)+n_{s u b}-1} \mathbf{w}_{i}^{H} \mathbf{H}^{H}[\nu] \mathbf{H}[\nu] \mathbf{w}_{i}+n_{s u b} N_{R} \sigma_{n}^{2},
$$

where $\nu_{0}(i)$ is the index of the first subcarrier in $i$ th subband. 
In this work, we mainly focus on the beam alignment problem for SU-MIMO transmission. Nevertheless, the proposed beam alignment framework can be adjusted to the multi-user case in a straightforward manner due to the sub-band-wise beam alignment setting. For the multiuser scenario, different users are assigned to different sub-bands such that the sub-band-wise beam alignment problem is reformulated to a multi-user beam alignment problem. This idea is similar to the frequency division multiple access in conventional communication systems. The only change here is the determination of the number of sub-bands, which should be chosen based on both the number of users and the number of transmit antennas.

\subsubsection{NLOS beam alignment}

In the NLOS scenario, the receive signal consists of several NLOS MPCs. To utilize the power of the NLOS com- ponents, the hierarchical k-means codebook described in Section 3 is implemented in this scenario. Due to the high frequency selectivity of the considered $\mathrm{THz}$ NLOS channel, even the frequency response of two neighbor- ing subcarrier may be quite distinct. Thus, the sub-band beam alignment as used for the LOS scenario is not con- sidered in the NLOS case. Instead, specific beams need to be aligned to each subcarrier used in an SC-FDMA system for a fully optimum performance. The BA problem for the $\nu \mathrm{t} \mathrm{h}$ subcarrier is equivalent to finding the beam code $\mathbf{w}_{\nu}^{*}$ from the hierarchical k-means codebook with maximal mean receive power for the $\nu \mathrm{t} \mathrm{h}$ subcarrier. Here, the mean receive power $P_{\nu}\left(\mathbf{w}_{\nu}\right)$ for precoding vector $\mathbf{w}_{\nu}$ and the $\nu \mathrm{t}$ h subcarrier is given by

$$
P_{\nu}\left(\mathbf{w}_{\nu}\right)=\mathbf{w}_{\nu}^{H} \mathbf{H}^{H}[\nu] \mathbf{H}[\nu] \mathbf{w}_{\nu}+N_{R} \sigma_{n}^{2} .
$$

Similar to the extension to multi-user transmission in the LOS case, the beam alignment framework for the NLOS scenario can be adjusted to a multi-user transmission as well. In the case of multi-user transmission, different users are assigned to different subcarriers such that the subcarrier-wise beam alignment algorithm is modified to a multi-user beam alignment algorithm.

\subsubsection{HBA problem formulation}

To accelerate the BA process in the large-scale MIMO case, we reformulate the BA problem to a stochastic MAB problem for stationary environments. The transmission system is considered as a time slotted system with $T$ time slots to search for the optimal beam. At the begining of the BA phase, the propagation scenario can be determined at the transmitter side based on the feedback of the receive power profile. If the propagation environment is an LOS scenario, the sub-band BA discussed in the LOS beam alignment is adopted for the following BA procedure. Otherwise, the subcarrier BA introduced in the NLOS beam alignment is utilized.

If an LOS component can be received, all $M$ subcarriers will be divided into $n_{s}$ sub-bands and each sub-band has to be aligned with an optimal beam. Thus, the BA problem is transformed to $n_{s}$ parallel BA problems, where $n_{s}$ is the number of sub-bands. For the $i$ th sub-band, we interpret its receive power as the measured reward $r_{i}$, i.e.,

$$
r_{i}=\sum_{\nu=\nu_{0}(i)}^{\nu_{0}(i)+n_{s u b}-1}\|\mathbf{r}[\nu]\|_{2}^{2}
$$

where $\mathbf{r}[\nu]$ is the receive signal vector for the $\nu$ th subcarrier. Consider the beam codes in the hiearchical DFT codebook as the arms in an MAB framework. Due to the randomness of data and noise, the reward $r_{i}$ is a random variable. This indicates that our LOS BA problem can be classified as a stochastic MAB problem. Assuming a stationary indoor scenario, we consider the channel as constant during the BA process. For simplicity, the reward is modeled as Gaussian distributed with variance $n_{s u b} \sigma^{2}$. The average payoff function $\mathbb{E}\left[r_{i}\left(\mathbf{w}_{i}\right)\right]$ for a beam code $\mathbf{w}_{i}$ in the hierarchical DFT codebook is equivalent to the average receive power $P_{i}\left(\mathbf{w}_{i}\right)$,

$$
\mathbb{E}\left[r_{i}\left(\mathbf{w}_{i}\right)\right]=P_{i}\left(\mathbf{w}_{i}\right),
$$

In the time slot $t$, the transmitter selects a beam code $\mathbf{w}_{i}(t)$ from the hierarchical DFT codebook $\mathbf{W}_{D F T}$ for the $i$ th sub-band. At the receiver, the power $r_{i}\left(\mathbf{w}_{i}(t)\right)$ of the $i$ th sub-band is measured and fed back to the transmitter. At the end of time slot $t$, the transmitter obtains the measured rewards of all sub-bands for this time slot and decides which arm to select for which sub-band based on a specific rule for the next time slot $t+1$.

For a stochastic MAB problem, the performance of the algorithm is evaluated via the expected cumulative regrets over $T$ time slots. Here, the expected cumulative regrets for the $i$ th sub-band $R_{i}(T)$ is defined as the expected difference between the cumulative reward of the optimal arm $\mathbf{w}_{i}^{*}$ and the cumulative reward of the proposed algorithm for $i$ th sub-band, given by

$$
R_{i}(T)=\sum_{t=1}^{T}\left(r_{i}\left(\mathbf{w}_{i}^{*}\right)-r_{i}\left(\mathbf{w}_{i}(t)\right)\right) .
$$

The objective of the design of the MAB algorithm is to find a selection policy that minimizes the sum expected cumulative regret $R_{L O S}(T)$ over all sub-bands, i.e., $R_{L O S}(T)=$ $\sum_{i=1}^{n_{s}} R_{i}(T)$.

The BA problem for the NLOS scenario is similar to that for the LOS scenario. Here, the BA problem can be transformed to $M$ parallel BA problem. For the $\nu$ th subcarrier, we interpret its receive power $\|\mathbf{r}[\nu]\|_{2}^{2}$ as the measured reward $r_{\nu}$, and consider the beam codes in the hierarchical $\mathrm{k}$-means codebook as the arms in an MAB framework. The reward is modeled as Gaussian distributed with variance $\sigma^{2}$. The average payoff function $\mathbb{E}\left[r_{\nu}\left(\mathbf{w}_{\nu}\right)\right]$ for beam code $\mathbf{w}_{\nu}$ is equivalent to the average receive power $P_{\nu}\left(\mathbf{w}_{\nu}\right)$,

$$
\mathbb{E}\left[r_{\nu}\left(\mathbf{w}_{\nu}\right)\right]=P_{\nu}\left(\mathbf{w}_{\nu}\right)=\sigma_{a}^{2} \mathbf{w}_{\nu}^{H} \mathbf{H}^{H}[\nu] \mathbf{H}[\nu] \mathbf{w}_{\nu}+N_{r} \sigma_{n}^{2} .
$$


In each time slot $t$, the transmitter selects beam codes for each subcarrier from the hierarchical k-means codebook. At the receiver, the receive power $r_{\nu}$ for the $\nu$ th subcarrier is measured and fed back to the transmitter. At the end of time slot $t$, the transmitter obtains the measured rewards of all subcarriers for this time slot and decides which arm to select for which subcarrier based on a specific rule for the next time slot $t+1$. In the NLOS scenario, the expected cumulative regrets $R_{\nu}(T)$ for $\nu$ th subcarrier is given by

$$
R_{\nu}(T)=\sum_{t=1}^{T}\left(r_{\nu}\left(\mathbf{w}_{\nu}^{*}\right)-r_{\nu}\left(\mathbf{w}_{\nu}(t)\right)\right)
$$

where $\mathbf{w}_{\nu}^{*}$ is the optimal beam code for the $\nu$ th subcarrier. The objective of the algorithm design for the NLOS case is to find a selection policy that minimizes the sum expected cumulative regret $R_{N L O S}(T)$ over all subcarriers, i.e., $R_{N L O S}(T)=\sum_{\nu=\nu_{0}}^{\nu_{0}+M-1} R_{\nu}(T)$.

\subsection{HBA procedure}

In the following, we extend the HBA algorithm proposed in [9] to the considered LOS and NLOS SC-FDMA transmission. As mentioned, the BA problems for both LOS and NLOS scenarios, respectively, can be decomposed into several independent sub-BA problems, which can be solved in parallel. The HBA algorithm discussed in the following provides an efficient approach to solve one independent BA problem, such as a sub-band BA problem in the LOS case and a subcarrier BA problem in the NLOS case, respectively. The procedure of the HBA algorithm is shown in Algorithm 2. The algorithm is designed based on the hierarchical structure of the average payoff function with respect to the code words. For a single BA problem, consider the beam code $\mathbf{w}(i, j)$ in the hierarchical codebook. If $\mathbf{w}(i, j)$ performs well, its left child $\mathbf{w}(i+1,2 j-1)$ and right child $\mathbf{w}(i+1,2 j)$ are highly likely to perform well, too. Hence, the algorithm will explore intensively within the beam coverage $C V(\mathbf{w}(i, j))$ with a good reward and loosely in others. For this proposal, a search tree is generated with nodes associated with the beam coverage, independently for each sub-BA problem. The node in a higher layer covers a smaller region as discussed in the codebook design part. The algorithm operates in discrete time slots, and in each time slot $t$, a new beam code is chosen by a deterministic rule based on the attributes of the search tree. The code selection procedure is executed independently for each sub-band or subcarrier in the LOS or NLOS scenario, respectively. After measurement at the receiver, the observed rewards will be fed back to the transmitter. Afterwards, the transmitter updates the attributes of the entire search tree based on the newly observed rewards $r^{t}$ and the newly selected code is added to the search tree. By repeating this selectionupdate process, the HBA algorithm narrows the searching region intelligently until a close-to-optimal beam code is selected.

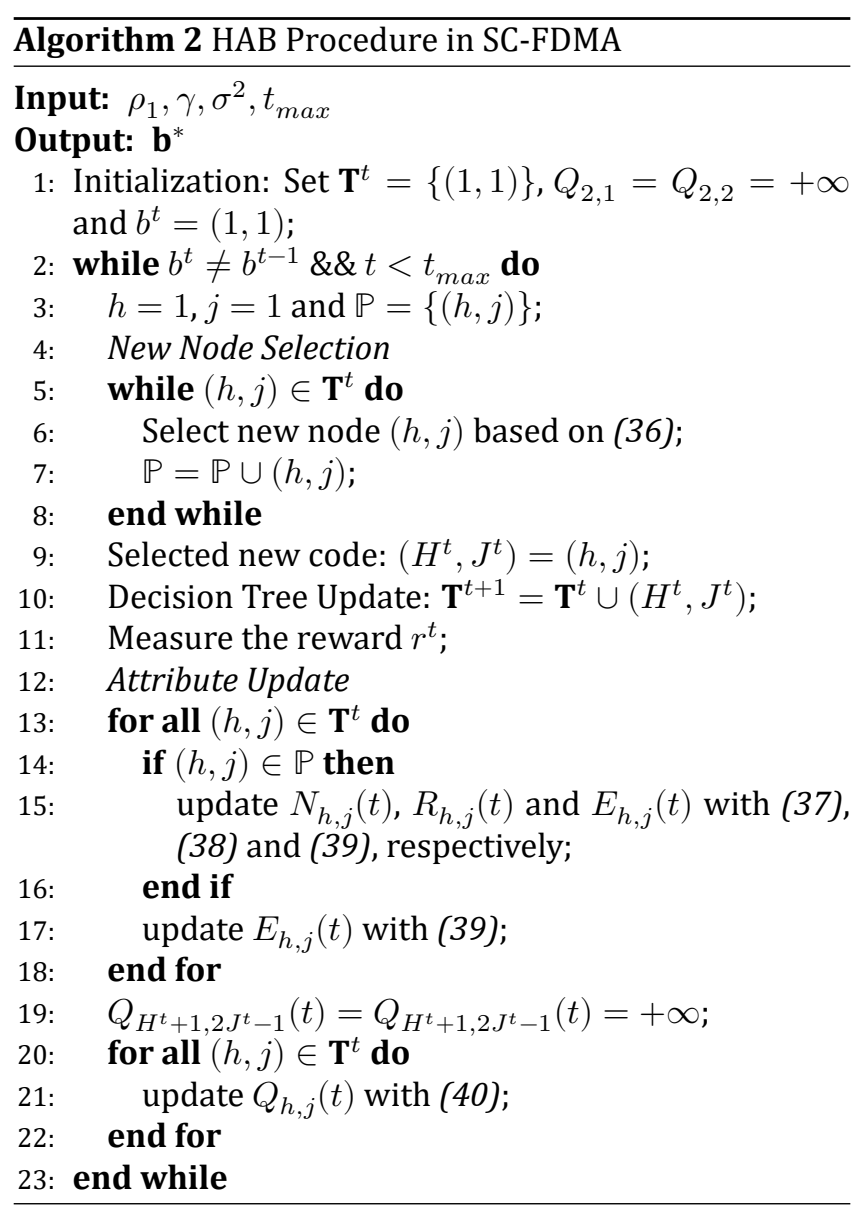

\section{New node selection}

In the new node selection phase, the HBA will select the beam code $\mathbf{w}^{t}$ with maximal estimated mean reward-Qvalue for one subcarrier or sub-band. The procedure of node selection can be found from line 3 to line 10 in Algorithm 2. By exploiting the hierarchical codebook structure, a binary tree search is implemented to find the new node. The binary search tree $\mathbf{T}$ is initialized in the beginning. A node in $\mathbf{T}$ is represented by $(h, j)$, where $h$ is the depth from the root node and $j, 1 \leq j \leq 2^{h-1}$ is the index at depth $h$. The corresponding beam code of node $(h, j)$ is $\mathbf{w}(h, j)$. After initialization, $\mathbf{T}^{1}$ contains only the root $(1,1)$. Assume $\mathbf{T}^{t}$ is the search tree at time $t$. Starting from the root node, the Q-values of two child nodes are compared until a new node $\left(H^{t}, J^{t}\right) \notin \mathbf{T}^{t}$ is selected. For a node $(h, j)$, the selection criterion is to choose its child $\left(h+1, j^{*}\right)$ with largest Q-value, that is:

$$
j^{*}= \begin{cases}2 j, & Q_{h+1,2 j}(t)>Q_{h+1,2 j-1}(t) \\ 2 j-1, & Q_{h+1,2 j}(t)<Q_{h+1,2 j-1}(t) \\ 2 j-\operatorname{Ber}(0.5), & Q_{h+1,2 j}(t)=Q_{h+1,2 j-1}(t)\end{cases}
$$

where $\operatorname{Ber}(0.5)$ represents a Bernoulli distributed random variable with a parameter of 0.5 . All selected nodes during the compare-select procedure are saved in $\mathcal{P}$, which is the path from the root node to the selected node. The finally selected node $\left(H^{t}, J^{t}\right)$ is added to the search tree $\mathbf{T}^{t}$ to obtain the search tree $\mathbf{T}^{t+1}$ for the next time slot $t+1, \mathbf{T}^{t+1}=\mathbf{T}^{t} \cup\left(H^{t}, J^{t}\right)$. 


\section{Attribute update}

In this step, the attributes of all nodes in the search tree $\mathbf{T}^{t+1}$ are updated based on the feedback of the measured reward $r^{t}$ in the current time slot. For LOS BA, $r^{t}$ is the measured receive power of one sub-band, while $r^{t}$ is the measured receive power of one subcarrier in the NLOS case. The details of the attribute update are shown in Algorithm 2 from line 12 to line 22. The update of $Q$-values consists of the following steps.

At first, the number of times $N_{h, j}(t)$ that node $(h, j)$ has been visited until time slot $t$ is updated as

$$
N_{h, j}(t)=N_{h, j}(t-1)+1, \forall(h, j) \in \mathcal{P}_{\nu} .
$$

Node $(h, j)$ must have been visited one time when it is selected as the new node for the search tree. $(h, j)$ will be visited one more time when one of its descendants is added to the search tree $\mathbf{T}^{t}$. The average measured reward $R_{h, j}$ of $(h, j)$ is updated by

$$
R_{h, j}(t)=\frac{\left(N_{h, j}(t)-1\right) R_{h, j}(t-1)+r^{t}}{N_{h, j}(t)}, \forall(h, j) \in \mathcal{P} .
$$

The empirical average reward $E_{h, j}(t)$ of node $(h, j)$ in time slot $t$ is defined as

$E_{h, j}(t)= \begin{cases}R_{h, j}(t)+\sqrt{\frac{2 \sigma^{2} \log t}{N_{h, j}(t)}}+\rho_{1} \gamma^{h}, & \text { if } N_{h, j}(t)>0 \\ +\infty, & \text { otherwise }\end{cases}$

where $\sqrt{\frac{2 \sigma^{2} \log t}{N_{h, j}(t)}}$ represents the confidence margin related to the uncertainty of rewards, related to random data and noise. With increasing $N_{h, j}(t)$, the uncertainty of the reward of $(h, j)$ becomes lower, since there are more available observations. The confidence margin is designed based on Bayesian principle and derived in [26]. Here, $0<\gamma<1$ and $\rho_{1}>0$ are parameters of the algorithm, and $\rho_{1} \gamma^{h}$ specifies the maximum variation of the average reward function within beam coverage $C V$ $(\mathbf{w}(h, j))$ [26], which depend on the codebook structure. The datails re- garding $\gamma$ and $\rho$ selection can be found in [26] and [9]. If $\gamma$ and $\rho$ is chosen based on the bounded diameter prin- ciple and well-shaped region principle from [9], HBA will converge to the optimal beam code with high probability. In the initial phase of the HBA, no information regarding the rewards is available. Hence, $E_{h, j}(t)$ is initialized by infinity. With abundant observed rewards within $C V(h, j)$ available, we can tighten the upperbound of mean rewards step by step. Finally, the estimated maximum mean reward $Q(h, j)$ in beam coverage $C V(h, j)$ is determined as [26]

$$
Q_{h, j}(t)=\left\{\begin{array}{l}
\min \left\{E_{h, j}(t),\right. \\
\left.\max \left\{Q_{h+1,2 j-1}(t), Q_{h+1,2 j}(t)\right\}\right\}, \\
\text { if } N_{h, j}(t)>0 \\
+\infty, \text { otherwise }
\end{array}\right.
$$

When the HBA algorithm has obtained a sufficient number of observed rewards within the searching tree, it will
Table 1 - SC-FDMA system setting

\begin{tabular}{l|l} 
Parameter & Numerical Value \\
\hline DFT size $M$ & 1200 \\
DFT size $N$ & 2048 \\
Cyclic prefix length $L_{c}$ & 512 \\
Transmission band & $0.1-0.1281 \mathrm{THz}$ \\
Number of transmit antennas $N_{t}$ & 64 \\
Number of receive antennas $N_{r}$ & 2 \\
Antenna gain $G_{t}, G_{R}, G_{t}=G_{r}$ & $20 \mathrm{dBi}$ \\
Signal constellation & $4 \mathrm{QAM}$
\end{tabular}

narrow its searching coverage in the highest layer. The algorithm will be terminated, if no new node has been selected and the selection result no longer changes, i.e.,

$$
\mathbf{T}^{t+1}=\mathbf{T}^{t} .
$$

Then, the currently selected beam $\mathbf{w}\left(H^{t}, J^{t}\right)$ is the derived beam for the correponding sub-band and subcarrier in LOS and NLOS cases, respectively. According to [9], the computational complexity of the HBA is quadratic in the number of processed time slots, $O\left(T^{2}\right)$.

\subsection{Complexity analysis}

At time slot $T$, for one subcarrier or sub-band, the decision tree contains $T$ nodes as the tree is extended by one node in each time slot. The attributes of all nodes in a decision tree should be updated in each time slot, and hence the run time in time slot $T$ is linear in $T$, i.e., $O(T)$. As the algorithm is executed for $T$ time slots, the total computational complexity of the proposed HBA algorithm is quadratic in $T$, i.e., $O\left(T^{2}\right)$ [9].

\section{NUMERICAL RESULTS}

In the following, we investigate a THz SC-FDMA system, whose parameter settings are provided in Table 1. The transmission scenario is the indoor scenario considered in [6]. The transmitter is fixed at the center of the room ceiling and the location of the receiver with fixed height $h=1.5 \mathrm{~m}$ is uniformly distributed within the indoor environment. The results are averaged over 500 channel realizations. The proposed algorithm is compared to the following benchmarks:

Optimal SC-FDMA beamforming: In this beamforming scheme, the CSI is considered as known at both the receiver and the transmitter. Thus, an MMSE frequency domain equalizer according to [10] can be designed. This algorithm aims to minimize the MSE after equalization, which can be formulated as a convex optimization problem. The optimal solution is derived in [10], and its performance can be regarded as a performance upper bound for our proposed scheme.

Random beamforming: In random beamforming, the beamforming vector for each subcarrier or sub-band is a random complex vector with constant $L_{2}$-norm and random phase profile. An MMSE equalizer is employed at the 


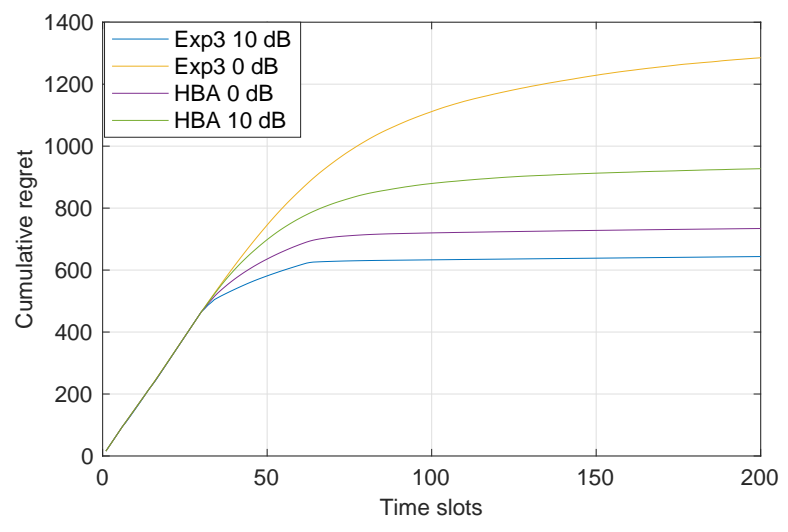

Fig. 7 - Cumulative regret of different algorithms in LOS scenario.

receiver as well. This scheme is related to a performance lower bound.

Exhaustive search: Exhaustive search is a naive beam alignment approach. In this scheme, the transmitter applies all beam codes from the predefined codebook several times to obtain the rewards of all beam codes [9]. Then the beam code with the largest measured reward is selected for usage. This beamforming method ensures that the optimal beam code from the available codebook is always obtained. Its performance serves for quantifying the loss due to the beam misalignment caused by the HBA.

Exponential weights (Exp3) algorithm: The Exp3 algorithm is based on the adversarial MAB framework. In [16], the authors advocated applying the Exp3 algorithm to the beam alignment problem. Compared to HBA, the Exp3 algorithm does not take the hierarchical structure of the codebook into account, which results in a slow convergence behavior for the beam code selection. Its convergence behavior can be taken as a reference for the convergence behavior of the HBA.

\subsection{Cumulative regret}

Figures 7 and 8 depict the sum cumulative regret $R(T)$ performance of the proposed HBA algorithm in LOS and NLOS scenarios, respectively, where the curves have been averaged over the receiver locations. Here, SNR is defined as the transmit power of one antenna divided by the noise power at one receive antenna. First, a bounded regret behavior is observed for both LOS and NLOS scenarios, which complies with the conclusion from [9]. In addition, the cumulative regret and the noise power are positively correlated, which indicates that the HBA needs more time slots to converge to the optimal beam under low SNR. However, under all SNR conditions, the HBA can achieve nearly $100 \%$ beam accuracy after 40 time slots, as confirmed by the bounded regret behavior. Moreover, under all SNR conditions in both LOS and NLOS scenarios, HBA performs better than Exp3 with respect to cumulative regrets. This is due to the fact that HBA utilizes the hierarchical structure of the codebook. Thus, searching

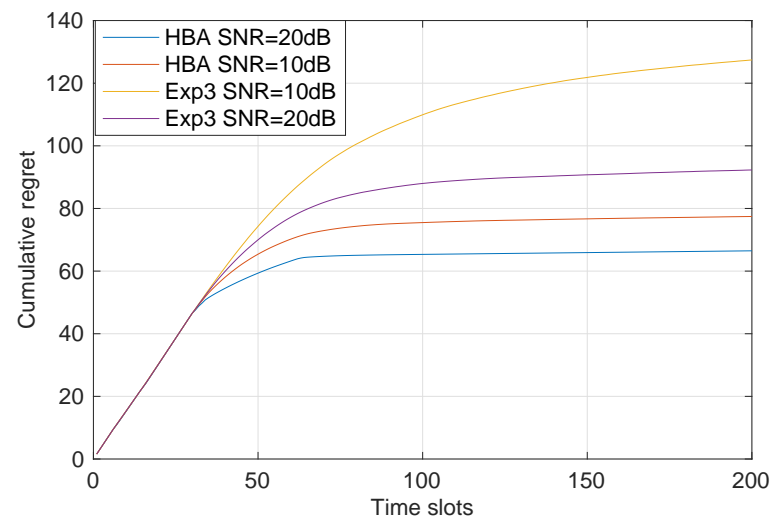

Fig. 8 - Cumulative regret of different algorithms in NLOS scenario.

over the entire codebook is avoided in HBA. Meanwhile, Exp3 operates like a random searching in the beginning, which results in a large number of time slots to converge.

\subsection{Convergence behavior}

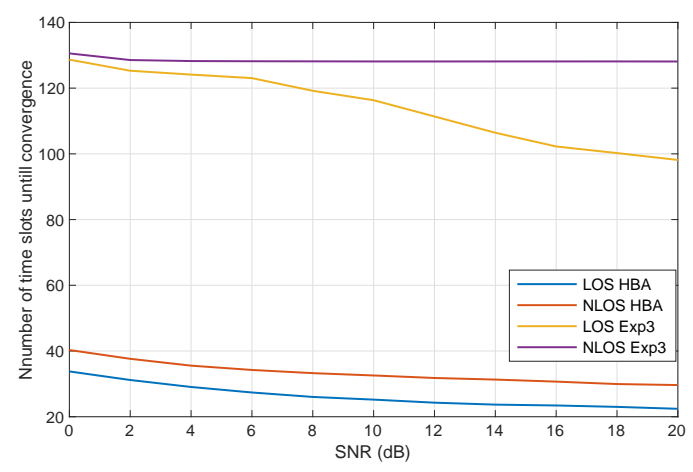

Fig. 9 - Convergence behavior of different BA schemes in both LOS and NLOS scenario.

Figure 9 illustrates the convergence behavior of the HBA in both LOS and NLOS scenario. In high SNR conditions, only 30 time slots are required for convergence to the finally selected beam, which is much faster compared to the Exp3 algorithm, requiring nearly 100 time slots to converge [9]. Furthermore, the convergence speed is related to the SNR, i.e., the HBA needs more time slots to converge to the optimal beam under low SNR, suggested also by Fig. 9. The reason is that in low SNR, the measured rewards are severely affected by the noise and the HBA requires more feedback information from the receiver to determine the mean reward. In all SNR conditions, our proposed approach converges faster compared to the benchmark schemes.

\subsection{BER performance}

In Fig. 10, the BER performance of the different beamforming schemes is shown for the LOS scenario. Apparently, the performance of the HBA is significantly better than that of random beamforming. Furthermore, the HBA 


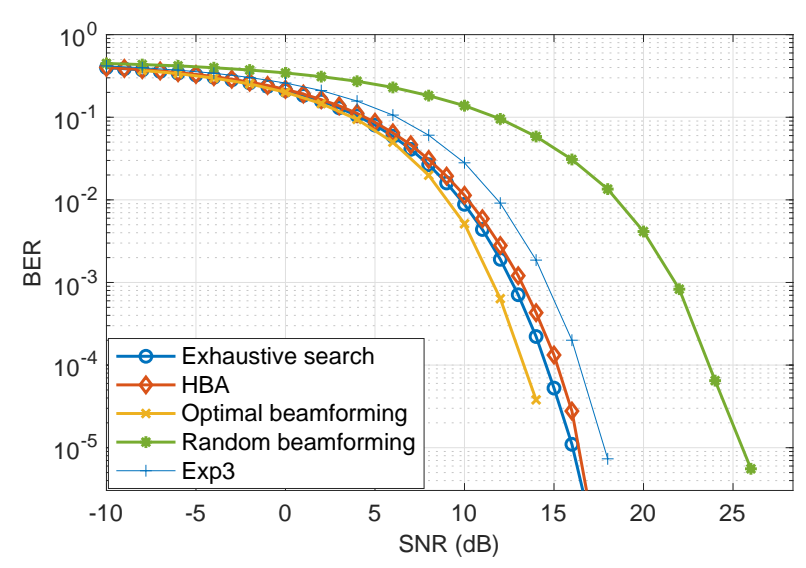

Fig. 10 - Average BER vs. SNR for different beamforming schemes in LOS scenario.

achieves a performance close to that of the optimal beamforming and exhaustive search, respectively, which complies with Theorem 2 and demonstrates the benefits of the HBA. In addition, although the Exp3 algorithm can achieve a similar BER performance as the HBA, Exp3 requires double the number of the time slots than HBA. Hence, HBA is able to provide a close-to-optimal beam selection with significantly shorter latency in the LOS scenario compared to the benchmark schemes.

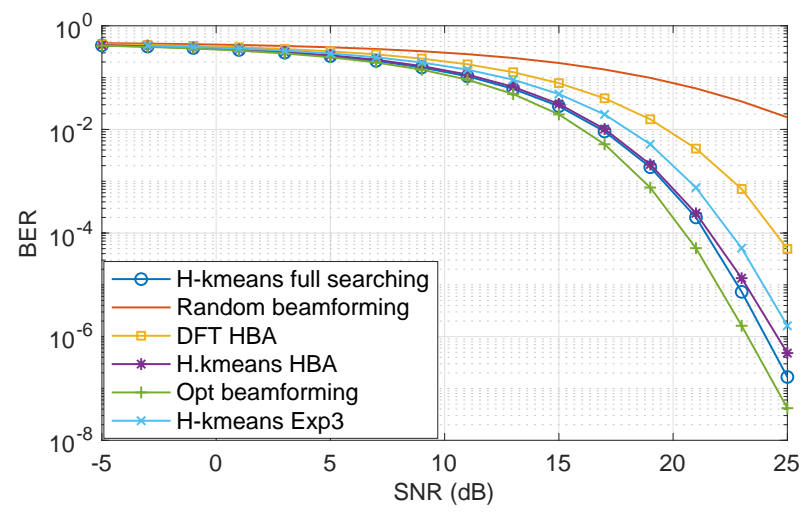

Fig. 11 - Average BER vs. SNR for different beamforming schemes in NLOS scenario.

In Fig. 11, the BER performance for the NLOS scenario is shown. To illustrate the performance of a hierarchical k-means codebook, the performance of the HBA with hierarchical DFT codebook is shown in addition as a benchmark. First, HBA with hierarchical k-means codebook design can improve the system performance by $3 \mathrm{~dB}$ compared to the HBA with DFT codebook. The HBA algorithm in the NLOS scenario achieves a performance close to the performance upper bound as well. The gap between the proposed scheme and optimal beamforming is reduced to less than $1 \mathrm{~dB}$. Thus, the hierarchical k-means codebook design can better exploit the NLOS components compared to the DFT codebook. We can also state that HBA with hierarchical k-means codebook is more likely to converge to a suboptimal beam code than HBA in the LOS case, according to the gap between exhaustive search and HBA.
The reason is that the NLOS channel suffers from strong frequency-selectivity. Thus, the training channels within the same beam coverage may have a large distance, which might direct the HBA to a non-optimal code. Besides, the reduced channel quality in the NLOS case increases the misalignment rate significantly. However, this performance degradation is less than $1 \mathrm{~dB}$, which is acceptable compared to the high exploration cost for the exhaustive search.

\section{CONCLUSION}

In this paper, hierarchical beam alignment with hierarchical codebook design for SU-MIMO THz communications has been studied. First, the hierarchical codebook design problem in MIMO THz communications has been established. Next, the hierarchical codebooks for LOS and NLOS propagation have been designed based on DFT codebook and data-driven hierarchical k-means clustering, respectively. Then, the beam alignment problem in $\mathrm{THz}$ communications has been formulated and the HBA from mmWave communications is adjusted to the SC-FDMA SU-MIMO THz communication system. Numerical results show that HBA combined with hierarchical DFT codebook can achieve a performance close to the optimal beamforming from [10] in a LOS scenario, while in an NLOS scenario HBA combined with hierarchical k-means codebook outperforms the DFT codebook. In our future work, the HBA will be extended to a multi-user transmis- sion.

\section{APPENDIX A}

\section{Proof of Theorem 1}

Here, the maximum over the codebook can be replaced by the $L_{\infty}$-norm as

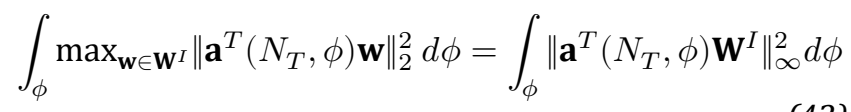

Regarding the optimization problem in (14), the constraints can be relaxed to a convex constraint, i.e., $\mathbf{w}_{j}^{H} \mathbf{w}_{j} \leq 1,1 \leq j \leq 2^{i-1}$, resulting in

$$
\begin{aligned}
& \max _{\mathbf{W}} \int\left\|\mathbf{a}^{T}\left(N_{T}, \phi\right) \mathbf{W}\right\|_{\infty}^{2} \mathrm{~d} \phi \\
& \quad \text { s.t. } \mathbf{w}_{j}^{H} \mathbf{w}_{j} \leq 1, i=1,2, \cdots, N_{T}, 1 \leq j \leq N_{T} .
\end{aligned}
$$

$\mathbf{F}$ is a local optimum for (12), if and only if there exists a Lagrange multiplier vector $\lambda$ guarantees the KKT conditions are satisfied. The Lagrangian of (12) is given by

$$
\hat{G}(\mathbf{W}, \lambda)=\int_{\phi}\left\|\mathbf{a}^{T}\left(N_{t}, \phi\right) \mathbf{W}\right\|_{\infty}^{2} d \phi-\sum_{i=1}^{N_{T}} \lambda_{j}\left(\mathbf{w}_{j}^{H} \mathbf{w}_{j}-1\right) .
$$


The gradient of Lagrangian with respect to $F_{i, k}^{*}$ is given by

$$
\begin{aligned}
\frac{d \hat{G}}{d F_{i, k}^{*}}= & \frac{d \int_{0}^{2 \pi}\left(\left\|\mathbf{F a}\left(N_{t}, \phi\right)\right\|_{\infty}^{2}\right) d \phi}{d F_{i, k}^{*}}-\lambda_{k} F_{i, k} \\
= & \int_{\frac{2 i-1}{N_{T}} \pi}^{\frac{2 i+1}{N_{T}} \pi} \frac{d\left|\sum_{l=0}^{N_{T}-1} F_{i, l} e^{j(l-1) \phi}\right|^{2}}{d F_{i, k}^{*}} d \phi-\lambda_{k} F_{i, k} \\
= & \int_{\frac{2 i-1}{N_{T}} \pi}^{\frac{2 i+1}{N_{T}} \pi} \sum_{l=0}^{N_{T}-1} e^{\frac{-j 2 \pi i l}{N_{T}}} e^{j \phi(l-k)} d \phi-\lambda_{k} F_{i, k} \\
= & e^{\frac{-j 2 \pi i k}{N_{T}}} \sum_{l=0}^{N_{T}-1} e^{\frac{-j 2 \pi i(l-k)}{N_{T}}} \frac{e^{\frac{(l-k)(2 i+1)}{N_{T}}}-e^{\frac{(l-k)(2 i-1)}{N_{T}}}}{j(i-k)} \\
= & e^{-\lambda_{k} F_{i, k}} \sum_{l=0}^{\frac{-j 2 \pi i k}{N_{T}}} \frac{2 \sin ((l-k) \pi)}{l-k}-\lambda_{k} F_{i, k} \\
= & \hat{D}_{i, k} F_{i, k}-\lambda_{k} F_{i, k},
\end{aligned}
$$

where $\hat{D}_{i, k}=\sum_{l=0}^{N_{T}-1} \frac{2 \sin ((l-k) \pi)}{l-k}$, which is not dependent on $i$. Therefore, there exists a Lagrange multiplier $\lambda$ that satisfies the KKT conditions. Hence, $\mathbf{F}$ is a local optimum for the optimization problem (14) and (42).

\section{APPENDIX B}

\section{Proof of Theorem 2}

Proof. According to [16], the receive power $\left|\mathbf{a}_{s}^{H}\left(N_{t}, \Phi\right) \mathbf{a}_{s}\left(N_{t}, \Phi_{i, j}\right)\right|^{2}$ is given by

$$
\left|\mathbf{a}_{s}^{H}\left(N_{t}, \Phi\right) \mathbf{a}_{s}\left(N_{t}, \Phi_{i, j}\right)\right|^{2}=\frac{\sin ^{2}\left(N_{t}\left(\Phi_{i, j}-\Phi\right)\right)}{\sin ^{2}\left(\Phi_{i, j}-\Phi\right)}
$$

Inserting $\Phi_{i, j}=\frac{\pi(2 j-1) 2^{I-i}}{N_{t}}$ to the above equation, we obtain:

$$
\begin{aligned}
\frac{\sin ^{2}\left(N_{t}\left(\Phi_{i, j}-\Phi\right)\right)}{\sin ^{2}\left(\left(\Phi_{i, j}-\Phi\right)\right)} & =\frac{\sin ^{2}\left(N_{t}\left(\frac{\pi(2 j-1) 2^{I-i}}{N_{t}}-\Phi\right)\right)}{\sin ^{2}\left(\Phi_{i, j}-\Phi\right)} \\
& =\frac{\sin ^{2}\left(N_{t} \Phi\right)}{\sin ^{2}\left(\Phi_{i, j}-\Phi\right)}
\end{aligned}
$$

Hence, maximizing the receive power $\left|\mathbf{a}_{s}^{H}\left(N_{t}, \Phi\right) \mathbf{a}_{s}\left(N_{t}, \Phi_{i, j}\right)\right|^{2}$ over the codebook in the $i^{\text {th }}$ layer is equivalent to finding the minimum of $\sin ^{2}\left(\Phi_{i, j}-\Phi\right)$, which corresponds to minimizing $\left|\Phi_{i, j}-\Phi\right|$ with $1 \leq j \leq 2^{i-1}$. In other words, the beam coverage of $\mathbf{w}(i, j)$ is a set of the steering vector with LOS spatial angle close to $\Phi_{i, j}$. Since the distance between $\Phi_{i, j}$ and $\Phi_{i, j-1}$ is $\frac{4 \pi}{2^{i}}$, the beam coverage of $\mathbf{w}(i, j)$ is obtained as

$$
C V(\mathbf{w}(i, j))=\left[\Phi_{i, j}-\frac{2 \pi}{2^{i}}, \Phi_{i, j}+\frac{2 \pi}{2^{i}}\right] .
$$

Next, $N_{t}$ is set to $2^{I}$. By inserting $N_{t}=2^{I}$ and $\Phi_{i, j}=$ $\frac{\pi(2 j-1) 2^{I-i}}{N_{t}}$ into the above equation, the result in (17) can be obtained.

\section{APPENDIX C}

\section{Proof of Theorem 3}

The objective function in (22) can be reformulated as

$$
\begin{aligned}
& \sum_{i=1}^{N_{p}} \sum_{\nu=\nu_{0}}^{\nu_{0}+M-1} \max _{\mathbf{w}_{j} \in \mathbf{W}}\left\|\mathbf{H}_{N L O S}^{\nu, i} \mathbf{w}_{j}\right\|_{2}^{2} \\
= & \sum_{i=1}^{N_{p}} \sum_{\nu=\nu_{0}}^{\nu_{0}+M-1} \max _{\mathbf{w}_{j} \in \mathbf{W}} \operatorname{tr}\left(\left(\mathbf{H}_{N L O S}^{\nu, i}\right)^{H} \mathbf{H}_{N L O S}^{\nu, i} \mathbf{w}_{j} \mathbf{w}_{j}^{H}\right) \\
= & \sum_{i=1}^{N_{p}} \sum_{\nu=\nu_{0}}^{\nu_{0}+M-1} \max _{\mathbf{w}_{j} \in \mathbf{W}} \\
& -\frac{1}{2}\left(\operatorname{tr}\left(\left(\hat{\mathbf{H}}_{\nu, i}-\hat{\mathbf{W}}_{j}\right)\left(\hat{\mathbf{H}}_{\nu, i}-\hat{\mathbf{W}}_{j}\right)^{H}\right)\right) \\
& +\frac{1}{2} \operatorname{tr}\left(\hat{\mathbf{H}}_{\nu, i} \hat{\mathbf{H}}_{\nu, i}^{H}\right)+\frac{1}{2} \operatorname{tr}\left(\hat{\mathbf{W}}_{j} \hat{\mathbf{W}}_{j}^{H}\right),
\end{aligned}
$$

where $\hat{\mathbf{H}}_{\nu, i}$ and $\hat{\mathbf{W}}_{j}$ are defined as $\left(\mathbf{H}_{N L O S}^{\nu, i}\right)^{H} \mathbf{H}_{N L O S}^{\nu, i}$ and $\mathbf{w}_{j} \mathbf{w}_{j}^{H}$, respectively. Since $\operatorname{tr}\left(\hat{\mathbf{H}}_{\nu, i} \hat{\mathbf{H}}_{\nu, i}^{H}\right)$ and $\operatorname{tr}\left(\hat{\mathbf{W}}_{j} \hat{\mathbf{W}}_{j}^{H}\right)$ are constant, the optimization problem in (22) is equivalent to

$$
\min _{\mathbf{W}} \sum_{i=1}^{N_{p}} \sum_{\nu=\nu_{0}}^{\nu_{0}+M-1} \min _{\mathbf{w}_{j} \in \mathbf{W}} D\left(\hat{\mathbf{H}}_{\nu, i}, \hat{\mathbf{W}}_{j}\right)
$$

\section{APPENDIX D}

\section{Proof of Theorem 4}

Since $\operatorname{rank}(\mathbf{W})$ is fixed to $1, \mathbf{W}$ can always be decomposed as

$$
\mathbf{W}=\mathbf{w}_{x} \mathbf{w}_{x}^{H} .
$$

Hence, the optimization problem in (26) is equivalent to

$$
\begin{aligned}
\max _{\mathbf{w}_{x}} & \sum_{\mathbf{H} \in \mathcal{H}_{j}} \mathbf{w}_{x}^{H} \mathbf{H} \mathbf{w}_{x} \\
\text { s.t. } & \mathbf{w}_{x}^{H} \mathbf{w}_{x}=1 .
\end{aligned}
$$

The optimal solution for $\mathbf{w}_{x}$ is given by $\mathbf{w}_{\max }$, where $\mathbf{w}_{\text {max }}$ is the eigenvector of $\sum_{\mathbf{H} \in \mathcal{H}_{j}} \mathbf{H}$ corresponding to its largest eigenvalue.

\section{REFERENCES}

[1] Ian F Akyildiz, Josep Miquel Jornet, and Chong Han. "Terahertz band: Next frontier for wireless communications". In: Physical Communication 12 (2014), pp. 16-32. 
[2] Shahid Mumtaz, Josep Miquel Jornet, Jocelyn Aulin, Wolfgang $\mathrm{H}$ Gerstacker, Xiaodai Dong, and Bo Ai. "Terahertz communication for vehicular networks". In: IEEE Transactions on Vehicular Technology 66.7 (2017).

[3] Thomas Kürner. "Towards future THz communications systems". In: Terahertz science and technology 5.1 (2012), pp. 11-17.

[4] Zhi Chen, Xinying Ma, Bo Zhang, Yaxin Zhang, Zhongqian Niu, Ningyuan Kuang, Wenjie Chen, Lingxiang Li, and Shaoqian Li. "A survey on terahertz communications". In: China Communications 16.2 (2019), pp. 1-35.

[5] Viktoria Schram, Yifei Wu, Monika Kolleshi, and Wolfgang Gerstacker. "Comparison of Transmission Concepts for Indoor THz Communication Systems". In: 2020 Third International Workshop on Mobile Terahertz Systems (IWMTS). IEEE. 2020, pp. 1-5.

[6] Anamaria Moldovan, Michael A Ruder, Ian F Akyildiz, and Wolfgang $\mathrm{H}$ Gerstacker. "LOS and NLOS channel modeling for terahertz wireless communication with scattered rays". In: 2014 IEEE Globecom Workshops (GC Wkshps). IEEE. 2014, pp. 388-392.

[7] Hyung G Myung and David J Goodman. Single carrier FDMA: a new air interface for long term evolution. Vol. 8. John Wiley \& Sons, 2008.

[8] Chong Han, A Ozan Bicen, and Ian F Akyildiz. "Multi-ray channel modeling and wideband characterization for wireless communications in the terahertz band". In: IEEE Transactions on Wireless Communications 14.5 (2014), pp. 24022412.

[9] Wen Wu, Nan Cheng, Ning Zhang, Peng Yang, Weihua Zhuang, and Xuemin Shen. "Fast mmWave beam alignment via correlated bandit learning". In: IEEE Transactions on Wireless Communications 18.12 (2019), pp. 5894-5908.

[10] Uyen Ly Dang, Michael A Ruder, Robert Schober, and Wolfgang H Gerstacker. "MMSE beamforming for SC-FDMA transmission over MIMO ISI channels". In: EURASIP Journal on Advances in Signal Processing 2011 (2011), pp. 1-11.

[11] Viktoria Schram, Anamaria Moldovan, and Wolfgang $\mathrm{H}$ Gerstacker. "Compressive sensing for indoor THz channel estimation". In: 2018 52nd Asilomar Conference on Signals, Systems, and Computers. IEEE. 2018, pp. 1539-1546.

[12] Chunxiao Jiang, Haijun Zhang, Yong Ren, Zhu Han, Kwang-Cheng Chen, and Lajos Hanzo. "Machine learning paradigms for next-generation wireless networks". In: IEEE Wireless Communications 24.2 (2016), pp. 98-105.
[13] Nikhil Gulati and Kapil R Dandekar. "Learning state selection for reconfigurable antennas: A multiarmed bandit approach". In: IEEE Transactions on Antennas and Propagation 62.3 (2013), pp. 10271038.

[14] Morteza Hashemi, Ashutosh Sabharwal, C Emre Koksal, and Ness B Shroff. "Efficient beam alignment in millimeter wave systems using contextual bandits". In: IEEE INFOCOM 2018-IEEE Conference on Computer Communications. IEEE. 2018, pp. 2393-2401.

[15] Jianjun Zhang, Yongming Huang, Yu Zhou, and Xiaohu You. "Beam Alignment and Tracking for Millimeter Wave Communications via Bandit Learning". In: IEEE Transactions on Communications (2020).

[16] Irched Chafaa, E Veronica Belmega, and Mérouane Debbah. "Adversarial multi-armed bandit for mmwave beam alignment with one-bit feedback". In: Proceedings of the 12th EAI International Conference on Performance Evaluation Methodologies and Tools. 2019, pp. 23-30.

[17] Jianhua Mo, Boon Loong Ng, Sanghyun Chang, Pengda Huang, Mandar N Kulkarni, Ahmad AlAmmouri, Jianzhong Charlie Zhang, Jeongheum Lee, and Won-Joon Choi. "Beam codebook design for 5G mmWave terminals". In: IEEE Access 7 (2019), pp. 98387-98404.

[18] Ken Kreutz-Delgado. "The complex gradient operator and the CR-calculus". In: arXiv preprint arXiv:0906.4835 (2009).

[19] Radoslaw Piesiewicz, Christian Jansen, Daniel Mittleman, Thomas Kleine-Ostmann, Martin Koch, and Thomas Kurner. "Scattering analysis for the modeling of $\mathrm{THz}$ communication systems". In: IEEE Trans- actions on Antennas and Propagation 55.11 (2007), pp. 3002-3009.

[20] Sebastian Priebe and Thomas Kurner. "Stochastic modeling of $\mathrm{THz}$ indoor radio channels". In: IEEE Transactions on Wireless Communications 12.9 (2013), pp. 4445-4455.

[21] Zhengqing Yun, Zhijun Zhang, and Magdy F Iskander. "A ray-tracing method based on the triangular grid approach and application to propagation prediction in urban environments". In: IEEE Transactions on Antennas and Propagation 50.5 (2002), pp. 750-758.

[22] Y. Chen, Y. Li, C. Han, Z. Yu, and G. Wang. "Channel Measurement and Ray-Tracing-Statistical Hybrid Modeling for Low-Terahertz Indoor Communications". In: arXiv e-prints (2021). 
[23] Cen Lin and Geoffrey Ye Li. "Indoor terahertz communications: How many antenna arrays are needed?" In: IEEE Transactions on Wireless Communications 14.6 (2015), pp.3097-3107.

[24] Laura Pometcu and Raffaele D’Errico. “Channel Model Characteristics in D-Band for NLOS Indoor Scenarios". In: 2019 13th European Conference on Antennas and Propagation (EuCAP). IEEE. 2019, pp. 1-4.

[25] Zhenyu Xiao, Tong He, Pengfei Xia, and Xiang-Gen Xia. "Hierarchical codebook design for beamforming training in millimeter-wave communication". In: IEEE Transactions on Wireless Communications 15.5 (2016), pp. 3380-3392.

[26] Sébastien Bubeck, Gilles Stoltz, Csaba Szepesvári, and Rémi Munos. "Online optimization in X-armed bandits". In: Advances in Neural Information Processing Systems. 2009, pp. 201-208.

\section{AUTHORS}

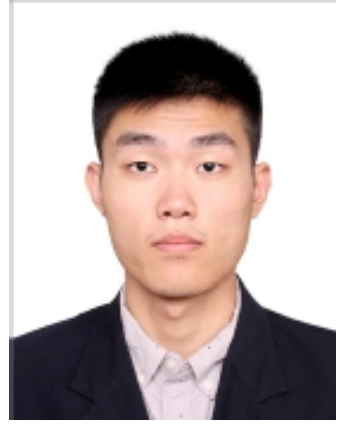

Yifei $\mathbf{W u}$ received the B.Eng. degree in automation from Zhejiang University, Hangzhou, China, in 2017, and the M.S. degree in electrical engineering from Friedrich-AlexanderUniversität Erlangen-Nürnberg, Erlangen, Germany, in 2020, where he is currently pursuing the Ph.D. degree. His research

interests include terahertz communication, intelligent reflecting surface-assisted communications, and convex optimization.

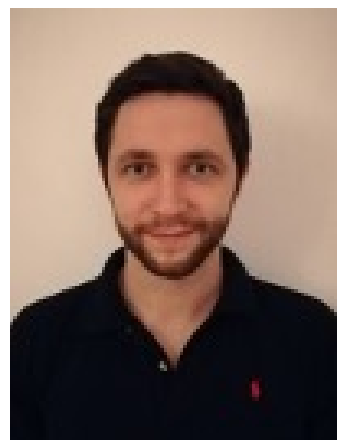

Johannes Koch was born in Bochum, Germany, in 1992. He received the M.Sc. degree in electronic engineering from the Friedrich-AlexanderUniversität Erlangen-Nürnberg (FAU), Erlangen, Germany in 2018, where he is currently pursuing the PH.D. degree. In 2018 he joined the Institute for Microwaves and Photonics (LHFT), FAU. His current research interests include THz communication systems, $\mathrm{THz}$ channel measurements and channel modeling above $100 \mathrm{GHz}$.

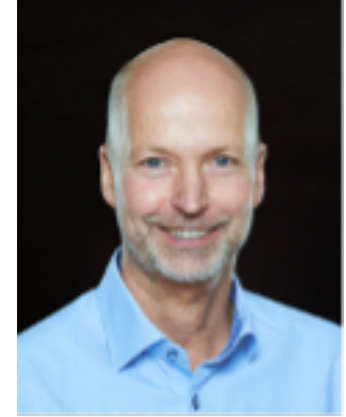

Martin Vossiek received the Ph.D. degree from RuhrUniversität Bochum, Bochum Germany, in 1996. In 1996, he joined Siemens Corporate Technology, Munich, Germany, where he was the Head of the Microwave Systems Group, from 2000 to 2003. Since 2003, he has been a Full Professor with

Clausthal University, Clausthal-Zellerfeld, Germany. Since 2011, he has been the Chair of the Institute of Microwaves and Photonics (LHFT), FriedrichAlexander-Universität Erlangen-Nürnberg (FAU), Erlangen, Germany. He has authored or coau- thored more than 300 articles. His research has led to over 90 granted patents. His current research in- terests include radar, transponder, RF identification, communication, and wireless locating systems. Martin Vossiek is a member of the German National Academy of Science and Engineering (acatech) and of the German research foundation (DFG) review board. He is a member of the German IEEE Microwave Theory and Techniques (MTT)/ Antennas and Propagation (AP) Chapter Executive Board and a member of the IEEE MTT Technical Committees MTT-24 Microwave/mm-wave Radar, Sensing, and Array Systems; MTT-27 Connected and Autonomous Systems (as founding chair); MTT-29 Microwave Aerospace Systems. He is also serving on the advisory board of the IEEE CRFID Technical Committee on Motion Capture \& Localization. Martin Vossiek has received more than 10 best paper prices and several other awards. For example, he was awarded the 2019 Microwave Application Award from the IEEE MTT Soci- ety (MTT-S) for Pioneering Research in Wireless Local Positioning Systems. Dr. Vossiek has been a member of organizing committees and technical program com- mittees for many international conferences and he has served on the Review Boards for numerous technical journals. From 2013 to 2019, he was an Associate Editor for the IEEE TRANSACTIONS ON MTT. 


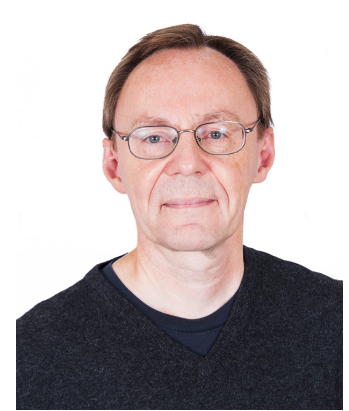

Wolfgang

Gerstacker received the Dipl.-Ing. degree in electrical engineering and the Dr.-Ing. and Habilitation degrees from the FriedrichAlexander University (FAU) Erlangen-Nü rnberg (FAU), Erlangen, Germany, in 1991, 1998, and 2004, respectively.

Since 2002, he has been with the Chair of Mobile Communications and Institute for Digital Communications, FAU Erlangen-Nürnberg, where he is currently a Professor. His research interests include the broad areas of digital communications and statistical signal processing, THz communications, 5G and Beyond, and wireless sensor networks. He has conducted various projects with partners from industry.

Dr. Gerstacker was a recipient of several awards, including the Research Award of the German Society for Information Technology (ITG) in 2001, the EEEfCOM Innovation Award in 2003, the Vodafone Innovation Award in 2004, the Best Paper Award of EURASIP Signal Processing in 2006, and the "Mobile Satellite \& Positioning" Track $\mathrm{Pa}$ - per Award of VTC2011-Spring. He has been the Technical Program Co-Chair or General Co-Chair of several confer- ences including BlackSeaCom 2014, VTC2013-Fall, ACM NanoCom 2016, and BalkanCom 2019. He is a member of the Executive Editorial Committee of the IEEE TRANS- ACTIONS ON WIRELESS COMMUNICATIONS and an Edi- tor of Computer Networks (Elsevier). He has served as a member of the Editorial Board for IEEE TRANSACTIONS ON WIRELESS COMMUNICATIONS, Physical Communica- tion (Elsevier), and EURASIP Journal on Wireless Commu- nications and Networking, and as a Guest Editor for sev- eral journals and magazines including the IEEE JOURNAL ON SELECTED AREAS IN COMMUNICATIONS, IEEE COMMUNICATIONS MAGAZINE, and IEEE TRANSACTIONS ON VEHICULAR TECHNOLOGY. 